
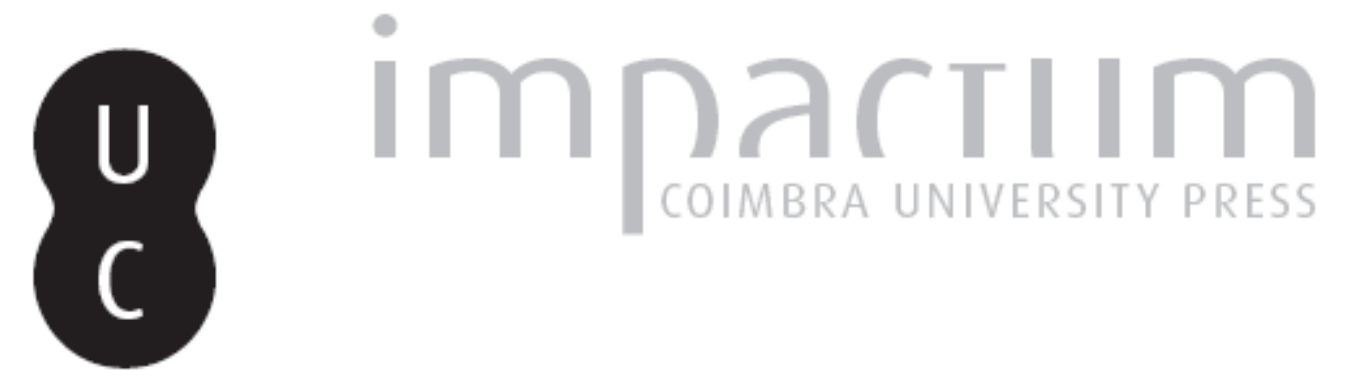

\title{
Production de l'huile et du vin en Lusitanie romaine
}

\section{Autor(es): Brun, Jean-Pierre}

Publicado por: Imprensa da Universidade de Coimbra

URL persistente:

URI:http://hdl.handle.net/10316.2/45428

DOI:

DOI:https://dx.doi.org/10.14195/1647-8657_36_3

Accessed : $\quad$ 26-Apr-2023 12:42:07

A navegação consulta e descarregamento dos títulos inseridos nas Bibliotecas Digitais UC Digitalis, UC Pombalina e UC Impactum, pressupõem a aceitação plena e sem reservas dos Termos e Condições de Uso destas Bibliotecas Digitais, disponíveis em https://digitalis.uc.pt/pt-pt/termos.

Conforme exposto nos referidos Termos e Condições de Uso, o descarregamento de títulos de acesso restrito requer uma licença válida de autorização devendo o utilizador aceder ao(s) documento(s) a partir de um endereço de IP da instituição detentora da supramencionada licença.

Ao utilizador é apenas permitido o descarregamento para uso pessoal, pelo que o emprego do(s) título(s) descarregado(s) para outro fim, designadamente comercial, carece de autorização do respetivo autor ou editor da obra.

Na medida em que todas as obras da UC Digitalis se encontram protegidas pelo Código do Direito de Autor e Direitos Conexos e demais legislação aplicável, toda a cópia, parcial ou total, deste documento, nos casos em que é legalmente admitida, deverá conter ou fazer-se acompanhar por este aviso.

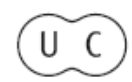


UNIVERSIDADE DE COIMBRA

FACULDADE DE LETRAS

\section{CONIMBRIGA}

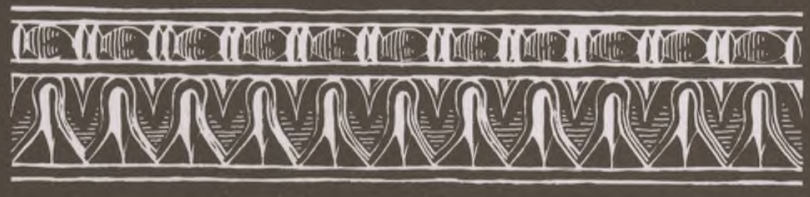

VOLUME XXXVI - 1997 


\section{JEAN-PIERRE BRUN}

CNRS, UMR 6573 Centre Camille Jullian et Etudes d'Antiquités A fricaines.

\section{PRODUCTION DE L'HUILE ET DU VIN EN LUSITANIE ROMAINE}

"Conimbriga" XXXVI (1997) p. 45-72

RESUMO: Procura fazer-se o ponto da situação acerca do que se conhece da produção de vinho e de azeite na Lusitânia romana.

Examinam-se os diferentes tipos de fontes - literárias, iconográficas e arqueológicas - com particular atenção para estas últimas, acentuando quão grandes são, ainda, as lacunas existentes.

A multiplicidade de estudos sobre as ânforas, cujo material permitiu que se conservassem até aos nossos dias, tem contribuído para ocultar a importância dos contentores de madeira (tonéis e pipas), sobrevalorizando-se a produção vinícola.

Por outro lado, o interesse que, desde há muito, vêm despertando as ricas villae romanas e os seus mosaicos levou a que se deixassem para um plano secundário as instalações de produção, que permanecem, por isso, insuficientemente conhecidas. Uma breve análise do que actualmente se sabe - mormente em relação às villae de $\mathrm{S}$. Cucufate, Torre de Palma e Freiría (em Cascais) - possibilita, no entanto, que se proponham algumas grandes linhas de evolução.

A oleicultura e a vinicultura desenvolvem-se desde o século I, como se conclui dalgumas instalações de produção e do aparecimento de certos tipos de ânforas vinárias. O auge da produção, no século II, está, em nosso entender, dissimulado pela utilização crescente - e bem depressa exclusiva - de utensílios de madeira: prensas, pipas, tonéis. A continuidade da viticultura no Baixo Império encontra-se atestada pela presença de lagares em vários grandes domínios, entre os quais o de Torre de Palma, que pensamos poder interpretar como lagar de vinho. Os vestígios arqueológicos documentam, além disso, a evolução das técnicas: difusão rápida dos lagares de vara e parafuso; substituição dos dolia por tonéis, do século II em diante, por influência, nomeadamente, 
das técnicas célticas. O oleicultura permanece, porém, menos conhecida, devido à raridade dos vestígios claramente interpretáveis.

PALAVRAS-CHAVE: Lusitânia, época romana, viticultura, oleicultura, lagar, tonel, ânfora, villa.

RÉSUMÉ: Cet article tente de présenter l'état des connaissances sur la production du vin et de l'huile en Lusitanie à l'époque romaine. Les différents types de sources, textuelles, iconographiques et archéologiques sont examinés en mettant l'accent sur ces dernières et en cernant l'ampleur de nos lacunes pour tenter une restitution des "chaînons manquants". Par exemple, la multiplication des études sur les amphores dont le matériau permet la conservation occulte l'importance des contenants en bois (tonneaux et foudres) et donc survalorise la production des sauces de poisson, certes considérable, au détriment de la production de vin. Par ailleurs, l'intérêt porté depuis longtemps aux riches villae romaines et à leurs mosaïques a joué au détriment des installations de production qui restent mal connues. Un bref examen des installations connues à ce jour, et notamment celles des villae de São Cucufate, de Torre de Palma, de Freiria (Cascais), permet toutefois de proposer quelques grandes lignes d'évolution. L'oléiculture et la viticulture se développent dès le $1^{\text {er }}$ siècle comme le montrent quelques installations de production et l'apparition de certains types d'amphores vinaires. L'essor de la production au II siècle est, selon nous, masqué par l'utilisation croissante et bientôt exclusive d'instruments en bois: presses, foudres, tonneaux. Le maintien de la viticulture au BasEmpire est attesté par la présence de pressoirs dans plusieurs grands domaines dont celui de Torre de Palma que nous proposons d'interpréter comme un pressoir à vin. Les vestiges archéologiques nous renseignent aussi sur l'évolution des techniques: rapide diffusion des pressoirs à levier et vis, remplacement des dolia par des tonneaux dès le II ${ }^{e}$ siècle sous l'influence des techniques celtiques, par exemple. L'oléiculture reste plus mal connue du fait de la rareté des vestiges clairement interprétables.

Mots-CLEFS: Lusitanie, époque romaine, viticulture, oléiculture, pressoir, tonneau, amphore, villa. 


\section{PRODUCTION DE L'HUILE ET DU VIN EN LUSITANIE ROMAINE (0}

Traiter de l'oléiculture et de la viticulture en Lusitanie, sur le territoire du Portugal d'aujourd'hui( $\left.{ }^{2}\right)$, revient à inventorier nos lacunes: presque aucune source écrite, des vestiges archéologiques encore rares et d'interprétation difficile, une iconographie intéressante mais que l'on doit se garder de surinterpréter. Comme presque partout dans le monde romain, toute la documentation écrite a disparu. L'exemple de l'Egypte romaine doit constamment éveiller notre vigilance sur ce point: les activités agricoles dont nous allons examiner quelques traces s'intégraient dans une organisation complexe où les acteurs économiques (état, cités, producteurs, marchands, clients) agissaient dans le cadre d'une législation, d'une fiscalité et de contrats de droit privé. Toutes ces relations étaient matérialisées par une abondante "paperasse" seulement conservée grâce au climat très sec de la vallée du Nil et du Fayoun: cadastres, contrats de vente de domaines, de vignobles, voire de récoltes sur pied, contrats de location de main d'oeuvre agricole, d'huilerie, de parcelles de terre, contrats de fabrication de conteneurs en céramique,

(') Il me faut remercier ici Pierre André, qui m'a fait redécouvrir le Portugal au cours d'un mémorable voyage d'étude en novembre 1996, Jorge de Alarcão qui a bien voulu accueillir cet article dans la revue Conimbriga et Adília Moutinho Alarcão qui, non contente d'encourager la rédaction de cette mise au point, a toujours répondu avec gentillesse à mes incessantes demandes de documentation.

Mes remerciements vont aussi à G. Cardoso et $\mathrm{J}$. d'Encarnação qui ont bien voulu m'autoriser à publier une photographie et un plan de l'installation de pressurage qu'ils ont mis au jour dans la villa de Freiría.

(2) Par commodité, nous incluons dans cette étude quelques gisements portugais situés au nord du Douro (Lavra, Fonte do Milho et Tralhariz) et n'appartenant pas à la Lusitanie romaine mais à la Galice.

Conimbriga, 36 (1997) 45-72 
en bois, en cuir pour le traitement et la vente de l'huile et du vin, contrats de transports, rôles d'impôts, reçus de prêts, etc. Pour la Lusitanie, comme partout ailleurs en Europe, il ne nous reste que des mentions fugitives dans des oeuvres littéraires ou documentaires et les vestiges archéologiques, encore trop peu exploités.

\section{Les sources}

\subsection{Les textes}

Il y a peu à tirer des textes antiques sur l'économie agricole de la Lusitanie. Pour la période qui précède immédiatement la conquête romaine, nous disposons du témoignage de Polybe (XXXIV, 8 apud Athénée Vili, 330c), qui visita la région en 151 avant J.-C. Il nous apprend que la Lusitanie est fertile et cite les prix de divers produits agricoles, parmi lesquels le vin ("un métrète (39 litres) de vin coûte une drachme"). Etant donné le contexte et le prix relativement bas, il semble clair qu'il s'agit de vin local et non d'importation.

Pour la période romaine, le seul texte qui évoque la production du vin en Lusitanie est la Géographie de Strabon. Le livre III, qui traite de la Péninsule Ibérique, a été écrit en 18 après J.-C. et décrit la vallée du Tage: 'L'étier supérieur renferme une île longue d'environ 30 stades et d'une largeur à peine inférieure, couverte d'une végétation luxuriante et de belles vignes (sùàpnsXov). Cette île se trouve à la hauteur de Moro" (à proximité de Santarém) (III, 3, 1). L'insignifiance de ce renseignement contraste avec la description détaillée de la Turdétanie due au même auteur qui évoque notamment la qualité du vin et de l'huile produits en abondance dans la vallée du Baetis-Guadalquivir (III, 2, 6). Durant tout l'Empire, cette région sera vantée par les écrivains pour l'excellence de ses productions; son huile et son vin seront attestés par l'épigraphie sur pierre et sur d'innombrables amphores. L'absence de mention du vin de Lusitanie dans les textes littéraires semble indiquer que leur renommée n'atteignait pas les cercles dirigeants de Rome; mais cela n'implique pas que la production de vin et d'huile était négligeable et leur commerce seulement local. En tout cas, certaines olives lusitaniennes, les praedulces, qui, aux dires de Pline (N. H. XV, 17), étaient plus douces que du raisin sec, avaient acquis une réputation flatteuse. On les cultivaient seulement en Afrique 
et en Lusitanie, autour à'Emerita, mais le texte de Pline ne permet pas de savoir s'il faut prendre l'expression stricto sensu (autour de la ville, dans le territoire de la cité?) ou lato sensu (dans la province de Lusitanie, dont le nom à'Emerita serait emblématique).

Strabon (III, 3, 7) évoque également la vie des montagnards de l'intérieur de la Lusitanie: sobres, ils ne boivent généralement que de l'eau ou de la bière; le vin n'est consommé que lors de banquets familiaux. Il semblerait donc qu'aux alentours du changement d'ère, la vente du vin lusitanien chez les populations de l'intérieur était limitée (à moins qu'il ne s'agissse d'une version locale du topos du sobre montagnard); en tout état de cause, cette situation a pu rapidement évoluer.

\subsection{Les représentations figurées}

Les représentations dyonisiaques dans les mosaïques sont trop banales dans l'ensemble de l'Empire pour constituer autant d'indices d'une production locale de vin, même si, dans certains cas, il y a pu avoir une volonté d'évoquer une production prestigieuse du domaine: ce pourrait être éventuellement le cas de la mosaïque représentant Dionysos et la thiase bachique de Torre de Palma et de la mosaïque bachique de Santa Vitoria do Ameixial, villa dans laquelle on trouve aussi une invocation à Dionysos (Chaves 1956, p. 57). Il faut également rappeler que l'on peut voir à Mérida une mosaïque représentant trois vendangeurs foulant le raisin dans une cuve dont le déversoir donne sur une sorte de baquet de transport du moût (Freijeiro 1978). Enfin, Dionysos était honoré dans une villa au moins, celle d'Estoi à Milreu, où un buste de Dionysos couronné de pampres a été découvert au siècle dernier $\left(^{3}\right)$ et on sait désormais que cette grande exploitation possédait des vignes et des oliviers (Hanel 1989). Un tel culte n'est probablement pas dépourvu de signification: dans la province de Narbonnaise, par exemple, on connait désormais plusieurs cas d'association entre la production du vin et la présence de statues de Dionysos. Une enquête sur le Portugal permettrait, peut-être, d'associer d'autres sculptures dionysiaques à des villae.

(3) Matos 1995, p. 56-57. Une autre tête de Dionysos a été découverte à Mértola (Beja) hors contexte et plusieurs autres sont signalées sans provenance au Museu Nacional de Arqueologia (Matos 1995, p. 58-59 et 196-197).

Conimbriga, 36 (1997) 45-72 


\subsection{Les installations viticoles et oléicoles (fig. 1)}

Faute de document écrit, on souhaiterait pouvoir se fonder sur des données archéologiques. Les ruines des villae lusitaniennes sont célèbres et impressionnantes. Qu'il suffise de citer les villae de São Cucufate à Vila de Frades, d'Estoi à Milreu, de Torre de Palma à Monforte, parmi les plus connues. Toutefois, rares sont celles qui ont été totalement fouillées en accordant une attention suffisante aux vestiges agricoles. L'attrait des mosaïques historiées joue autant sur le chercheur que sur le grand public. Durant longtemps, on n'a cherché qu'à mettre au jour les parties d'habitation luxueusement décorées et on négligeait les bâtiments rustiques, aux sols de terre battue et au mobilier peu attirant. Dans la villa de Torre de Palma, la découverte des pressoirs par M. Heleno est la conséquence non d'un programme de recherche sur l'économie agricole, mais du dégagement quasi total des vestiges dû à d'autres objectifs; dans la villa de Santa Vitória do Ameixial, on a surtout dégagé les parties nobles, pièces de réceptions, thermes susceptibles de livrer des mosaïques (Chaves 1956). Aujourd'hui encore, les fouilles en cours des villae de Tourega (Évora) ou de Cegonha (Vidigueira) n'ont jusqu'ici mis au jour que les thermes, les parties d'habitation, les complexes religieux, alors que pour la première, au moins, un contrepoids de pressoir a été anciennement signalé (Gorges 1979, PS.26). En revanche, il faut mettre en exergue l'exemple remarquable des recherches conduites à l'occasion des fouilles de la villa de São Cucufate. Non seulement les fouilleurs se sont attachés à reconstituer les étapes de l'évolution de la villa, mais aussi à cerner l'ampleur de son domaine et la nature de ses productions, grâce au dégagement des parties agricoles et à des prospections systématiques dans son territoire (Alarcão, Etienne \& Mayet 1990). Toutefois, même dans ces quelques cas privilégiés, l'étude des productions agricoles n'est pas dénuée d'ambiguités: lorsque l'on découvre des pressoirs et des cuves, il n'est pas toujours aisé de distinguer s'il s'agit de vin ou d'huile. La présence de macro-restes végétaux, tels que pépins ou noyaux d'olives est un bon indice mais, dans les fouilles anciennes, ce type de vestige n'a que rarement été observé et sa mention est exceptionnelle: à Roxo (Santiago de Cacém), à la fin du siècle dernier, on a découvert un pressoir et des pépins de raisin (Alarcão, 1974, p. 47). Dans la villa de São Cucufate dont les fouilleurs ont estimé ne pas être en mesure de 
trancher entre l'huile et le vin $\left({ }^{4}\right)$, un certain nombre d'indices sont en faveur de ce dernier: des pépins de raisins ont été retrouvés dans la salle de manoeuvre des pressoirs; on ne trouve aucune trace de moulin à huile et bien des dolia étaient poissés (Alarcão, Etienne \& May et 1990, 246). Il existe des cas où les vestiges mis au jour sont sans ambiguïté: dans la villa de Ponte de Igreja (Nelas), un fouloir se déverse par une large goulotte dans une grande cuve: il semble clair que ce type d'installation, qui trouve de nombreux parallèles en Grèce, en Italie et en Gaule, était destiné à exprimer le jus de raisin comme l'avait bien vu Santos Rocha (1897) (fig. 2). Dans la villa de Torre de Palma, ce sont les particularités architecturales du bâtiment qui conduisent à s'interroger sur la destination d'un long bâtiment dans lequel se trouvent trois cuves et une énorme pierre de pressoir. Etant donné que ce secteur de l'Alentejo est, aujourd'hui, essentiellement voué à l'olivier, le pressoir a été attribué à l'extraction de l'huile d'olive (Sardinha d'Oliveira 1958). Or, malgré l'état d'arasement du site, nous verrons plus loin que les caractéristiques de l'édifice ne s'expliquent véritablement que s'il s'agit d'un chai à vin. En revanche, la dimension des cuves permet rarement une discrimination. Si les petites cuves jumellées sont attribuables à la décantation de l'huile, on trouve de grandes cuves aussi bien dans les installations viticoles qu'oléicoles (Brun 1993B). De même la présence d'une chaudière dans les pressoirs n'indique pas forcément qu'il s'agit d'une huilerie car on a autant besoin de feu pour faire réduire le moût afin de préparer le defrutum que pour chauffer l'eau destinée à faciliter l'extraction de l'huile.

Celle-ci n'est attestée avec certitude que lorsqu'on découvre de véritables moulins à huile formés d'une partie dormante circulaire dans laquelle tournent une ou deux meules cylindriques ou hémisphériques verticales. De tels moulins sont très rarement mentionnés dans la littérature archéologique: on ne connaît guère que celui de la villa de Fontão (Lavra), dans la région de Porto (Gorges 1979, p. 456). Une enquête sur le terrain permettrait certainement d'en inventorier d'autres, encore qu'en bien des endroits on devait utiliser un procédé que Columelle mentionne sous le nom de canalis et solea (XII, 52, 6). Il s'agit en fait d'une auge de bois inclinée dans laquelle un ouvrier chaussé de sabots de bois piétinait les olives. Ce type d'instrument, encore largement employé dans la vallée du Guadalquivir à l'époque moderne, ne laisse évidemment aucune trace archéologique.

(4) Mais, depuis la publication, Pierre Sillières a déterminé qu'il s'agissait d'une installation viticole (Leveau, Sillières et Vallat, 1993, p. 222). 


\subsection{Les amphores}

Peut-on compléter nos renseignements sur une éventuelle production locale de vin ou d'huile grâce à l'étude des amphores? On sait que les amphores étaient essentiellement utilisées pour le transport maritime des denrées liquides. A ce titre, des fabrications d'amphores à huile ou à vin au cours de l'Empire romain seraient de bons indices d'une production viticole ou oléicole assez importante pour être exportée. Or il faut reconnaître que, pour l'essentiel, les amphores lusitaniennes, entre le $\mathrm{I}^{\mathrm{er}}$ et le $\mathrm{V}^{\mathrm{e}}$ siècle, étaient destinées au transport des poissons conservés dans de la saumure et des sauces de poisson (le garum) (Mayet 1990). En marge de cette production omniprésente et écrasante, certains ateliers dans les vallées du Tage et du Sado ont peut-être produit des amphores vinaires. Ce serait le cas de l'atelier de Muge, dans la vallée du Tage, où l'on aurait fabriqué des amphores Dressel 2/4 au cours du Haut-Empire (Cardoso 1990, p. 156) et de celui de Porto dos Cacos, dans la basse vallée du Tage, qui fabriquait des amphores à fond plat (Raposo 1990, p.126-127). La production de cet atelier couvre les cinq premiers siècles de notre ère, mais ces amphores sont attribuables à une phase antérieure aux fours des IV-Ve siècles (Raposo \& Duarte 1996, p. 253). Mayet \& Tavares da Silva (1994a) et Mayet, Schmitt \& Tavares da Silva (1996, p. 22). interprètent ce type d'amphore comme une variante plus ancienne de l'amphore Almagro 51c et, donc, comme un récipient destiné au transport des salaisons, mais il paraît clair que cette amphore à fond plat dénommée Lusitana 3 imite tout autant les amphores de forme Gauloise 4, conteneur du vin de Narbonnaise, que de forme Dressel 30, conteneur du vin de Maurétanie Césarienne (Alarcào \& Mayet 1990, p. 187-189; Fabiâo 1996, p. 378-379) (fig. 3). Il est donc possible que les amphores de ce type produites à Porto dos Cacos étaient destinées au transport de vin local au cours des $\mathrm{II}^{\mathrm{e}}$ et $\mathrm{III}^{\mathrm{e}}$ siècles $\left.{ }^{5}\right)$. Le même type de production a été mis en évidence dans la vallée du Sado (Diogo \& Faria, 1990, fig. 52, n. ${ }^{\circ} 13$ et 53, n. ${ }^{\circ}$ 27), probablement à $\mathrm{Abul} \mathrm{A}\left({ }^{6}\right)$. Plusieurs exemplaires en ont

$\left.{ }^{5}\right)$ Diogo (1987, p. 185), signale également une possible production d'amphores imitant les Dressel 28 dans la vallée du Sado, mais cette indication demande à être étayée.

${ }^{6}$ ) Sur les amphores à fond plat d'Abul A : Mayet, Schmitt \& Tavares da Silva, 1996, p. 103. 
été trouvés en mer notamment dans l'embouchure du Tage, à Vila Franca de Xira, où il semble qu'une embarcation chargée d'amphores de ce type ait coulé (Diogo \& Alves 1988-1989).

En tout état de cause, aucun type d'amphore n'a pu jusqu'ici être attribué à l'huile de Lusitanie. Cela ne signifie pas pour autant qu'il n'y avait pas de production d'huile dans cette province, mais peut-être qu'elle n'était pas suffisante pour être exportée outre mer. Pour la consommation dans les villes lusitaniennes et pour le trafic intérieur, un transport d'huile dans des outres était plus adapté $\left({ }^{7}\right)$. En revanche, la Lusitanie importait de l'huile de Bétique: les amphores Dressel 20 ne sont pas rares sur le côtes lusitaniennes et même à l'intérieur des terres, y compris dans des villae qui ont pu produire de l'huile, notamment en Alentejo; c'est seulement dans le Nord du Portugal qu'elles se raréfient considérablement. Fabião (1993-1994) lie cette pénétration au grand trafic maritime qui transitait le long des côtes de la Lusitanie en direction de la Bretagne et aux approvisionnements officiels comme ceux de l'armée $\left(^{8}\right)$. Mais que signifient quelques amphores isolées ? De nombreuses régions oléicoles ont importé de l'huile de Bétique au cours du Haut-Empire: la Campanie (on connaît de nombreuses amphores Dressel 20 à Pompéi: Tchemia 1964), la Narbonnaise (large dispersion y compris dans les villae oléicoles: Brun 1986, p. 267-270). Sont-elles les témoins rescapés d'importations massives ou, au contraire, indiquent-elles des achats diversifiés normaux dans le cadre d'une économie ouverte ou bien encore sont-elles autant de preuves d'une économie administrée $\left({ }^{9}\right)$ ? Tel propriétaire a pu vouloir goûter la célèbre huile de Bétique, voire s'en procurer régulièrement, sans que cela soit interprétable comme le signe de l'absence de production d'huile lo-

(7) Il convient de remarquer à ce propos que la Lusitanie est voisine de régions climatiquement hostiles ou peu favorables à la culture de l'olivier comme la Galice et le nord-ouest de la Tarraconnaise. Il ne fait guère de doute que ces contrées montagneuses devaient être approvisionnées par portage d'outres.

$\left.{ }^{8}\right)$ Je ne pense pas que l'on puisse faire état dans cette problématique de l'inscription trouvée à Tocina, en Bétique, appartenant à un monument funéraire érigé par Marcus Cassius Sempronianus, un diffusor olearius originaire d'Olisipo (González Fernández 1983 et 1991, 2, n. ${ }^{\circ}$ 281). Certes ce négociant en gros était né à Olisipo mais rien ne permet de penser qu'il était impliqué dans le commerce de l'huile de Bétique vers la Lusitanie.

(9) Si l'on admet qu'une majorité de ces amphores proviennent bien de trafics officiels. 
cale $\left({ }^{10}\right)$. En fait, la situation était probablement complexe: des cargaisons ont dû être affrétées par l'administration, notamment l'armée, et parallèlement, souvent sur les mêmes bateaux une partie des amphores étaient destinées au commerce privé (c'est ce que Dick Whittaker (1989, p. 65) appelle joliment le "marché subventionné"). A côté de ces trafics, très visibles car on sait parfaitement reconnaître les Dressel 20, une production locale et un commerce interne ont pû fort bien prospérer et rester invisibles à nos yeux, l'une parce qu'on manque de fouilles et l'autre parce qu'on devait transporter l'huile dans des outres. En résumé, en dehors des phénomènes massifs, comme la production des amphores Dressel 14 et Almagro 51, qui témoignent d'une formidable production de salaisons de poissons, on a bien du mal à apprécier l'importance de la viticulture ou de l'oléiculture en fonction de la fabrication ou de l'importation de tel ou tel type d'amphore, car une part probablement considérable de l'huile et du vin était conditionnée dans des conteneurs en matériaux périssables. Les résultats de l'étude des amphores ne peuvent donc être la base unique de notre réflexion et ils doivent être relativisés par la prise en compte des autres sources.

\subsection{Les dolia}

Les viticulteurs italiens utilisaient de grandes jarres, appelées dolia, pour faire fermenter le jus de raisin. Ces dolia en céramique avaient des contenances variant entre 500 et 1500 litres en moyenne. Pour les rendre étanches et pour les stériliser, on les enduisait chaque année de poix, faite à partir de bois de résineux chauffés. En Italie et en Gaule méridionale, les installations viticoles romaines sont caractérisées par la présence de plusieurs dizaines à quelques centaines de ces

$\left({ }^{10}\right) \quad$ Il faut se garder de surinterpréter le témoignage des amphores surtout lorsque l'on traite des échantillons peu importants. Ainsi, la tentative de Pimenta (1982-1983) d'interpréter l'absence d'amphores vinaires importées après le Ir siècle de notre ère et celle d'amphores à huile après le II ${ }^{e}$ siècle comme le signe d'un essor de la production locale ne peut être prise en considération, car l'échantillon qu'il utilise ne comprend que 37 amphores provenant de plusieurs gisements de la région de Sintra et réparties sur cinq siècles. Dans un lot aussi petit, l'absence d'un type d'amphore n'a aucune signification. Une telle démarche n'aurait de validité que si l'on pouvait traiter plusieurs milliers d'amphores provenant de plusieurs gisements judicieusement répartis. 
jarres enterrées jusqu'au col dans le sol de hangars, les cellae vinariae. Il paraît probable que les chais à vin de Lusitanie en étaient également dotés, au moins au début de l'implantation romaine. En effet, les Italiens qui se sont installés dans cette région ont dû introduire leurs méthodes de culture et de vinification. La présence d'entrepôts contenant des dolia est donc un fort indice de production viticole. Il faut toutefois nuancer ce propos, car l'huile d'olive était également conservée dans des jarres, mais, pour des raisons évidentes de volume, ces dolia olearia sont toujours en petit nombre et peuvent être donc facilement distingués des jarres à vin. Des dolia contenant des traces de gras "fossilisées" auraient été anciennement découverts dans la villa de Alto da Fonte do Milho, à Canelas do Douro (région de Vila Real: Gorges 1979, p. 455); il faudrait toutefois pouvoir pratiquer des analyses en chromatographie pour obtenir une certitude. Par ailleurs, on a pu vinifier dans d'autres récipients que de jarres en terre-cuite, notamment dans des tonneaux de bois.

\subsection{Les tonneaux}

A Mérida, capitale de la Lusitanie, une stèle funéraire datée de la fin $d u$ Ier siècle ou du début du II ${ }^{e}$ siècle, représente un personnage ouvrant le robinet d'un tonneau. A partir de la fin du $\mathrm{II}^{\mathrm{e}}$ et au $\mathrm{III}^{\mathrm{e}}$ siècle, se répand la mode de se faire sculpter des tonneaux en ronde-bosse comme monument funéraire et d'y faire graver une épitaphe au milieu (fig. 4). Ce modèle de tombeau dérive de monuments en forme de demi-cylindres répandus en Lusitanie, en Tarraconnaise, en Afrique et en Maurétanie (Julia 1965). Leur signification est encore débattue, mais ceux de 1'Alentejo (cités d'Ebora, de Pax Iulia et Myrtilis) offrent des représentations très réalistes de tonneaux qui tranchent avec les autres (Julia 1965, pl. 18, 1; Matos 1995, p. 126-129). Il est possible qu'il s'agisse d'une interprétation locale d'un modèle dont la signification de départ était autre $(\mathrm{n})$. Alors que les inscriptions qui figurent sur les "cupae" stylisées du Sud du Portugal mentionnent surtout une onomastique servile, les "cupae" réalistes de l'Alentejo ont été comman-

(n) Julia (1965) a émis l'hypothèse que ce type de monument funéraire était originaire d'Afrique du Nord et qu'il s'était répandu au cours du II grands ports en relation avec l'Afrique. 
dées par des personnes libres — à consonnance latine, souvent des citoyens romains - et, exceptionnellement, par un indigène (Viana 1945; Encarnação 1984, p. 813). Même si on ne peut exclure qu'il s'agisse d'une version locale du culte salvateur de Dionysos ailleurs illustré par des sarcophages-lènoi portant des représentations de vendanges et de foulage $\left({ }^{12}\right)$, ces représentations réalistes pourraient être en rapport avec la viticulture locale à l'instar du tonneau représenté sur la stèle du vigneron L. Cantius Acutus d'Aquilée. Quelle que soit l'explication, ces "cupae" réalistes doivent attirer notre attention sur la large utilisation du tonneau dans cette région de peuplement celtique, au moins à partir du $\mathrm{II}^{\mathrm{e}}$ siècle. Dans son ouvrage sur Le Vin de VItalie romaine, A. Tchernia a montré qu'à partir des Antonins la place prise par le tonneau au détriment de l'amphore dans la commercialisation du vin faussait les estimations des courants commerciaux (Tchernia 1986, p. 285-292). Cela signifie que la relative rareté des amphores vinaires lusitaniennes n'implique pas la faiblesse de la production de vin, puisque le vin a certainement été commercialisé en tonneaux, au moins à partir du II ${ }^{\mathrm{e}}$ siècle et peut-être auparavant.

On peut ajouter que le tonneau complique l'interprétation des vestiges archéologiques des villae. En effet, si les viticulteurs lusitaniens utilisent massivement des tonneaux pour transporter leur vin à partir du Ile siècle, il faut en conclure qu'il emploient aussi probablement des foudres de bois pour vinifier. Or ces vaisseaux, parfois très grands - Strabon $(\mathrm{V}, 1,12)$ évoque des foudres grands comme des maisons en Italie du Nord - , ne laissent pas de traces archéologiques. Toute interprétation des vestiges archéologiques, surtout du Bas-Empire, devra donc tenir compte de la possible disparition de ces foudres.

\subsection{L'apport des études environnementales}

Beaucoup reste à faire pour appliquer à l'archéologie classique les méthodes employées en Préhistoire: analyses polliniques, carpologiques et anthracologiques. Bien souvent, les premières sont très décevantes. Les gisements en plein air, comme les villae, n'offrent que

(12) Notamment au musée de Lisbonne: sarcophage des vendanges de Vila Franca de Xira (Matos 1995, p. 100-101).

Conimbriga, 36 (1997) 45-72 
rarement des sédiments valablement analysables, faute de bonnes conditions de conservation. Les prélèvements carpologiques et anthracologiques sont plus aisés à réaliser, mais on manque de spécialistes pour multiplier les études. Aussi doit-on se contenter de quelques données éparses qui, de plus, concernent seulement la Protohistoire: les analyses polliniques effectuées à Alpiarça, dans la région de l'estuaire du Tage, montrent des occurrences significatives de pollens de vitis et d'olea au cours de l'Age du Fer (Leenwaarden \& Janssen 1985, p. 229). On sait qu'il est impossible de distinguer les pollens des espèces sauvages et cultivées et les pollens de vitis, évidemment sauvage, sont déjà présents dès la base des colonnes sédimentaires d'Alpiarça dans un paysage encore largement forestier vers 5000 BP. A partir du VIe siècle avant J.-C. (date radiocarbone $2590 \mathrm{BP}$ ), les pollens de vitis représentent $33 \%$ et ceux d'olea apparaissent. Ce serait en fait l'augmentation significative de son pourcentage corrélée au recul de la forêt $\left({ }^{13}\right)$ et à l'extension des surfaces cultivées qui serait le signe de la culture de la vigne. L'apparition à la même période des pollens d'olivier serait également à mettre en relation avec l'introduction de l'oléiculture.

Ces premières études sont très prometteuses, mais il faudrait multiplier les analyses polliniques sur les gisements datés de l'époque historique ou sur des colonnes sédimentaires comprenant des niveaux de l'Antiquité pour obtenir des données présentant une valeur statistique et susceptibles d'être mises en série ( $\left.{ }^{14}\right)$.

\section{Un essai d'histoire des techniques viticoles et oléicoles}

\subsection{Aux origines}

Si notre connaissance de l'oléiculture et de la viticulture romaines au Portugal est lacunaire, celle de la période préromaine l'est encore plus. On peut inférer du témoignage de Polybe qu'au $\mathrm{II}^{\mathrm{e}}$ siècle avant J.-C. des vignobles existaient déjà et que la production était suffisante

(,3) La vigne sauvage est une liane qui pousse naturellement dans les forêts du pourtour méditerranéen.

$\left.{ }^{14}\right) \quad$ Une étude tendant à corréler les données environnementales et archéologiques a été récemment publiée sur l'oléiculture antique en Provence et pourrait servir d'exemple: Leveau et alii 1991.

Conimbriga, 36 (1997) 45-72 
pour maintenir un prix de vente raisonnable. Mais depuis quand? Une viticulture et une oléiculture locales s'étaient-elles développées sous l'influence des Phéniciens présents dans l'embouchure du Sado depuis le VII siècle ? Les vestiges retrouvés jusqu'ici à Abul se limitent à un établissement commercial occupé du VII au Ve siècle avant J.-C. (Mayet \& Tavares da Silva 1994A); mais, à travers ces relations commerciales, de nouvelles cultures, comme celles de la vigne et de l'olivier, ont pu être transmises par les Phéniciens aux populations locales. Les premières analyses polliniques effectuées dans l'estuaire $\mathrm{du}$ Tage pourraient renforcer cette hypothèse. Elles montrent que la vigne sauvage poussait naturellement au Portugal mais que sa mise en culture n'eut lieu qu'au début de l'Age du Fer parallèlement à celle de l'olivier. La coöncidence chronologique est probablement significative. Quoiqu'il en soit, les populations celtiques qui occupaient la Lusitanie entre 1'Anas et le Tage buvaient à la fois du vin importé de Phénicie, de Grèce, puis d'Italie, du vin local et certainement aussi, comme leurs congénères de Gaule, de la cervoise qu'ils devaient conserver et transporter dans des futailles en bois.

\subsection{Au Haut-Empire: huile ou vin?}

Parmi de bien nombreux changements, la conquête romaine et la déduction de colonies amenèrent de nouvelles formes de culture et une nouvelle gestion des territoires. Les techniques agricoles nous restent quasiment inconnues à moins d'étendre à la Lusitanie ce que Columelle (IV, 14, 2 et XI, 2, 60) et Pline (N. H. XIV, 29-30, XVII, 170) nous apprennent de l'agriculture de la Bétique: des vignes basses, taillées en gobelet, que l'on déchaussait, voire irriguait et que l'on vendangeait en juillet. Les nouvelles propriétés furent mises en valeur par des agriculteurs habitant des fermes dont la grandeur et le luxe augmentèrent au cours du $\mathrm{I}^{\text {er }}$ siècle après J.-C. L'exemple le mieux connu est désormais celui de la villa de São Cucufate: une première ferme fut construite vers le milieu du Ier siècle de notre ère. Elle comportait quelques pièces d'habitation pour le propriétaire, un bâtiment "rustique" qui pourrait avoir servi de logement à un intendant et au personnel et des pièces à usage de stockage. Parmi celles-ci certaines sont qualifiées par les auteurs de la publication, de cellae oleariae et/ou vinariae à cause de la découverte dans ce secteur de nombreux fragments de dolia. Ces 
pièces ouvrent sur le "couloir" 10, qui comportait trois files de dolia enterrés; ces derniers furent arasés et recouverts d'un sol en béton ultérieurement (fig. 5). Les auteurs de la publication ont bien vu qu'il s'agissait d'un entrepôt probablement pour les liquides et ils observent que nombre de fragments de dolia retrouvés dans ce secteur étaient poissés (Alarcão, Etienne \& Mayet 1990, p. 54 et 245-246). Ce type d'entrepôt rappelle fortement les chais à vin d'Italie et de Narbonnaise et le "couloir" 10 a toutes chances d'avoir servi à la vinification. Si cette interprétation est juste, cela signifierait que, dès les débuts de la villa, le domaine fut en partie consacré à la culture de la vigne et que, dans cette seconde moitié du $\mathrm{I}^{\mathrm{er}}$ siècle de notre ère, on vinifiait dans les villae de l'Alentejo selon des techniques italiennes. D'autres villae viticoles sont probablement attestées par des celliers à dolia, voire par des accumulations de fragments de dolia. La villa de Tralhariz, Quinta da Ribeira (région de Bragança) comportait plusieurs dolia. Le site de Canelas do Douro, Alto da Fonte do Milho (région de Vila Real) possédait un fouloir-pressoir et des dolia dont certains portent des inscriptions peintes (15) (fig. 8). Dans la grande villa de Quinta de Marim (Olhão, région de Faro), trois dolia étaient encastrés dans un pavement (Santos Rocha 1895). Ces témoignages sont encore trop imprécis quant au nombre et la contenance des dolia et, sauf à São Cucufate, trop mal datés pour tirer des conclusions. Mais on peut émettre l'hypothèse qu'il $s$ 'agit d'installations viticoles des Ier $^{\text {et }} \mathrm{II}^{\mathrm{e}}$ siècles de notre ère $\left({ }^{16}\right)$.

(15) Sur Tralhariz, Quinta da Ribeira, nous ne possédons qu'une description sommaire de Severo (1900, p. 396) qui mentionne au moins six dolia qu'il attribue à un cellier. Sur Canelas do Douro, voir Russell Cortez 1949 et 1951, p. 66-71, ainsi que Gorges 1979, p. 457-458 et Alarcão 1988, II, 1, p. 29, n. ${ }^{\circ}$ 1/494 . Dans une villa fortifiée occupée du $\mathrm{I}^{\mathrm{er}}$ au $\mathrm{V}^{\mathrm{e}}$ siècle de notre ère, se trouvent un complexe thermal et des bâtiments agricoles, notamment une installation viticole. Elle comprenait un fouloir dont le fond était formé de deux dalles fortement inclinées vers un déversoir et limité par des murettes, ou plutôt des planches fixées dans des rainures. On pressait sur ces mêmes dalles à l'aide d'un levier qui était ancré dans un massif maçonné au nord et dont le guidage était assuré par deux montants de pierre fixés dans le mur nord du fouloir (fig. 8). Le moût se déversait dans un réceptable qui en fait devait contenir un baquet de transport en bois analogue à celui qui est représenté sur la mosaïque des vendanges de Mérida. Le cellier dont ne subsistaient que trois fonds de dolia in situ s'étendait au sud du fouloir.

(16) Un ensemble de pressurage attribué à la vinification a été récemment fouillé dans la villa de Las Musas (Arellano, en Navarre). Il comporte deux aires de presse, deux cuves et un vaste cellier. Ce dernier, divisé en deux nefs par une file de pilier),

Conimbriga, 36 (1997) 45-72 
Le cas de São Cucufate est plein d'enseignements quant à l'évolution de la vinification dans l'Alentejo au $\mathrm{II}^{\mathrm{e}}$ siècle. Sous le règne d'Hadrien, une nouvelle villa fut construite qui, tout en intégrant les structures anciennes, accroissait considérablement les surfaces bâties: construction d'un péristyle, des thermes, de logements pour le personnel et d'installations agricoles. Parmi celles-ci, on trouve un pressoir doté de deux presses à levier, se déversant dans une cuve latérale à l'est (Alarcão, Etienne \& Mayet 1990, p. 81-82) (fig. 6). Des amas de pépins de raisins ont été trouvés dans la fosse des contrepoids; cet indice précieux sur la nature de la production semble trouver confirmation grâce à l'étude des structures bâties: les deux cuves situées en bout du pressoir, au nord, ne peuvent avoir fonctionnées avec ce dernier, car leur fond est pratiquement au même niveau que celui du pressoir. Il faut donc imaginer qu'elles recueillaient un liquide provenant d'un fouloir situé au nord et à un niveau plus élevé (un mètre environ); l'arasement des vestiges a dû le faire disparaitre. On pourrait donc reconstituer une séquence complète de vinification: fouloir surélevé, deux cuveaux de recueil, deux pressoirs en contrebas et un cuveau de recueil du vin de presse. Une telle installation suppose une production relativement importante de vin, mais il faut donc repérer le lieu où l'on faisait fermenter le moût. Au nord des pressoirs se situe un entrepôt (n. $\left.{ }^{\circ} 1\right)$ dont la toiture était soutenue par un pilier central (fig.7). Cette pièce, qui a été trouvée vide à l'exception de foyers aménagés relativement tardifs, pourrait avoir abrité soit des dolia defossa, soit des foudres de bois dans lesquels on vinifiait $\left({ }^{17}\right)$. Si cette dernière hypothèse était retenue, on assisterait au

mesure $28 \mathrm{~m}$ par 7,10 m et contenait 34 dolia retrouvés vides et, pour une part, retournés (Mesquiriz 1993). L'installation est datée du Ier siècle et semble avoir fonctionné jusqu'à la fin du III ${ }^{\mathrm{e}}$ siècle, époque où ce secteur de la villa a été incendié. Si l'attribution de l'installation est confirmée (une base de moulin à huile a été également trouvé dans le cellier), cela signifierait qu'en Navarre aussi on vinifiait dans des dolia au Haut-Empire.

$\left({ }^{17}\right) \quad$ Alarcão, Etienne \& Mayet 1990, 80. De nombreux fragments de dolia et de couvercles ont été découverts dans cette zone, mais aucun n'était pas en place. Les dolia étaient-ils enterrés dans l'entrepôt 1 et auraient-ils été extraits seulement au BasEmpire pour être remplacés par des tonneaux ? Ou bien sont-ils issus de l'arasement des dolia du "couloir" 10 ? Contre la première hypothèse, on remarquera que les fouilleurs n'ont pas retrouvé de logements creusés dans le substrat. Mais il faut reconnaître que le bâtiment était très arasé et que dans l'angle sud-est, mieux conservé, ils ont découvert un amas significatif de fragments.

Conimbriga, 36 (1997) 45-72 
passage, dès le début du $\mathrm{II}^{\mathrm{e}}$ siècle, d'une vinification de type italique, en dolia, à une vinification en foudres, clairement influencée par les techniques celtiques.

Une autre grande villa largement dégagée mérite un bref examen: celle de Torre de Palma, à Monforte. Dans une récente publication, $\mathrm{S}$. Maloney et J. Haie réexaminent ce gisement anciennement fouillé par M. Heleno à la lumière des recherches conduites par l'Université de Louisville (Kentucky, USA) (Maloney et Haie 1996). La site a connu six phases principales de construction entre le milieu du Ier siècle (état I) et le Moyen-Age (état VI). Au cours de l'état II, vers la fin du Ier siècle ou au début $\mathrm{du} \mathrm{II}^{\mathrm{e}}$ siècle, on édifia une vaste ferme comportant une maison de maître, des thermes et des bâtiments d'exploitation et de logement de la main d'oeuvre disposés autour de deux cours. La villa semble alors produire essentiellement des céréales stockées dans des greniers dont les sous-sols sont renforcés de longuerines. Dans la seconde moitié du $\mathrm{III}^{\mathrm{e}}$ siècle et/ou au début du $\mathrm{IV}^{\mathrm{e}}$ siècle, les anciens bâtiments sont totalement transformés: une nouvelle pars urbana est construite, très vaste, centrée sur un péristyle et ornée de superbes mosaïques. Les bâtiments agricoles sont alors remodelés et, à proximité immédiate de la nouvelle pars urbana et de l'ancienne maison du maître, dans une cour, on bâtit un ensemble de bassins enduits de béton de tuileau que M. Heleno avait identifié comme un lavoir. S. Maloney et $\mathrm{J}$. Haie proposent d'attribuer ces ruines à une installation viticole sur la base de comparaisons avec des installations du XIX ${ }^{\mathrm{e}}$ siècle. Il est à craindre toutefois que l'on ne puisse pas utiliser ces vestiges pour notre propos, car, si le plan publié peut faire penser effectivement à un grand fouloir se déversant dans deux cuves de recueil, un examen des vestiges en place montre que les niveaux de sols sont quasiment horizontaux et qu'il est impossible d'utiliser cette installation pour produire du vin de la façon dont procédaient les anciens (voir Palladius, I, XVIII, 1, par exemple). En revanche, il pourrait s'agir de réservoirs d'eau dont l'emplacement, au contact de la maison de maître, serait plus adapté qu'une cuverie génératrice de nuisances.

Toutes les transformations des produits agricoles n'étaient pas concentrées dans les campagnes: une partie était réalisée dans les villes par de modestes propriétaires. A Conimbriga, un fouloir à vin a été mis au jour dans l'Insula du Vase Phallique (Alarcâo \& Etienne 1977, p. 161) (fig. 9). La datation de cette structure est large, entre le IIe et le Vème siècle, mais elle est très caractéristique. Les raisins étaient 
déversés dans le fouloir qui mesure $2,90 \mathrm{~m}$ de long par 1,70 $\mathrm{m}$ de large; ils étaient foulés au pied et le moût s'écoulait dans le cuveau $(1 \mathrm{~m} \mathrm{x}$ $0,90 \mathrm{~m} \times 0,70 \mathrm{~m})$ qui avait une contenance utile de 600 litres environ. La vinification s'opérait dans des récipients qui n'ont pas été retrouvés. Il est possible que, là aussi, on ait utilisé des foudres de bois qui n'auraient pas laissé de trace. De même, un contrepoids de pressoir a été découvert dans les fouilles anciennes; on ne peut l'attribuer à l'huile ou au vin mais il témoigne d'activités de transformations agricoles dans la ville même, comme cela est connu en bien des endroits (Volubilis, Munigua, etc...) (Alarcão 1977, p. 79).

Pour cette période, on ne connaît pas de pressoir attribuable avec certitude à l'extraction de l'huile. Les mêmes appareils étaient utilisés pour les deux produits et il faut disposer d'installations complètes et bien conservées pour pouvoir discriminer les deux productions. Pour tenter d'estimer la place de la production locale, il faut prendre en compte les traces d'importation.

\section{U évolution des pressoirs}

L'évolution technique des pressoirs est relativement bien connue dans l'ensemble de l'Empire et il semble que la Péninsule Ibérique ait été plus ouverte aux progrès que d'autres provinces comme l'Afrique. Au Haut-Empire, tant pour l'huile que pour le vin, on utilisait généralement des pressoirs à levier et câbles. Ce type de pressoir utilise un long tronc d'arbre très lourd que l'on actionne avec des câbles de cuir manoeuvré à l'aide d'un treuil fixé à un gros bloc de pierre parallélépipédique. Des blocs hors contexte de ce type ont été signalés à plusieurs reprises, notamment à Pedrões, Santiago (région de Setúbal: Faria \& Ferreira 1986, p. 50), mais seul celui de la villa de Freiría (Cascais) a été retrouvé in situ. Les fouilles en cours, conduites par G. Cardoso et J. d'Encarnação, ont dégagé la pars urbana décorée de mosaïques, des thermes et la pars rustica comportant un grenier et une installation de pressurage qui comprend un pressoir et deux cuves (fig. 10 et 11). A l'extrémité de la presse se trouve une fosse approximativement carrée dans laquelle se trouve un contrepoids parallélépipédique en pierre creusé de deux encoches latérales en queue d'aronde et d'une rainure axiale sur la face supérieure. Ces logements recevaient des montants de bois latéraux maintenus ensemble par une tringle horizontale et servant 
à fixer le rouleau d'un treuil que l'on actionnait avec des barres de bois. Le levier du pressoir (leprelum) était manoeuvré à l'aide de câbles enroulés autour du treuil. Le marc de raisin ou les olives écrasées étaient pressés sur un sol de béton de tuileau (opus signinum) recouvrant un affleurement de rocher aplani. Le liquide extrait était dirigé vers des cuves disposées latéralement et enduites de béton de tuileau. Elles comportaient des boudins d'étanchéité aux angles et un (ou deux) puisard pour recueillir les restes de liquide lors des vidanges ou nettoyages. L'état actuel des recherches ne permet pas de déterminer avec certitude si cette installation servait au pressurage de $\mathrm{P}$ huile ou du vin. Peut-être la découverte d'éléments caractéristiques comme une meule à huile ou des macrorestes significatifs permettra de trancher la question; la présence de deux ensembles de petites cuves est toutefois un indice en faveur de l'huile $\left({ }^{18}\right)$.

Le remplacement de ces pressoirs à câbles, peu pratiques et relativement dangereux par des pressoirs à vis est un question encore débattue (Brun 1993A). Les premières attestations de pressoirs à vis remontent à la fin du Ir siècle avant J.-C. et se diffusent, en Italie au moins, au cours du Ier siècle de notre ère. En Narbonnaise, ce n'est pas avant la fin du II ${ }^{\mathrm{e}}$ siècle qu'ils remplacent les pressoirs à câbles. En Afrique, il semble que l'on n'ait jamais utilisé des pressoirs à vis même à l'époque byzantine, mais en Maurétanie Tingitane, à Volubilis notamment, on décèle le changement au cours du $\mathrm{II}^{\mathrm{e}}$ siècle (Akerraz \& Lenoir 1981-1982). Dans la Péninsule Ibérique, les pressoirs à vis sont omniprésents au Bas-Empire, mais quand font-ils leur apparition? Une huilerie mise au jour dans les fouilles de Munigua (région de Sevilla) est datée de la première moitié du Ier siècle de notre ère et elle est dotée d'un pressoir à levier et vis (Hanel 1989). Dans la villa de São Cucufate, les deux contrepoids de pressoir à vis sont attribués à la phase II, celle de l'édification d'une nouvelle villa à péristyle au cours du second quart du $\mathrm{II}^{\mathrm{e}}$ siècle (Alarcâo, Etienne \& Mayet 1990, p. 81-82), mais il faut toutefois rester prudent car les pressoirs ont continué de fonctionner au IVe siècle et on a pu alors effectuer des transformations, l'adaptation de vis et le remplacement des contrepoids par exemple, sans que cela laisse forcément des traces datables. Dans la majorité des cas, en effet, les contrepoids cylindriques de vis sont en relation avec les états tardifs des villae. En

(18) Le seul indice que Ton peut éventuellement utiliser est la présence d'un cercle de pierres, en grande partie détruit et situé dans la salle des cuves : s'agit-il des vestiges du socle d'un moulin à huile? (Fig. 11 en bas). 
tout état de cause, les débuts de ces transformations semblent anciens et dès les $\mathrm{I}^{\text {er }}$ et $\mathrm{II}^{\mathrm{e}}$ siècles, certaines installations sont dotés de pressoirs dernier cri. Ces nouveaux pressoirs employaient des vis de fort diamètre assujettis à des contrepoids de pierre cylindriques. La vis traversait l'extrémité du prelum et s'engageait dans un écrou fixé au dessus du levier. La vis était maintenue dans un logement grâce à une gorge enserrée par deux demi-plateaux solidement fixés dans le bloc de pierre par des tenons en queue d'aronde et de crochets de fer.

Si l'on connaît relativement bien le système de manoeuvre des pressoirs lusitaniens, pour l'essentiel des vis assujetties à des contrepoids, on ne sait quasiment rien de leur mode d'ancrage. Le pressoir de Sobões da Mina (Bensafrim dans 1'Algarve), mal daté, offre un bon exemple d'appareil rupestre dont le levier est encastré dans une paroi rocheuse (Santos Rocha 1896). Une mortaise permet de glisser une aiguille afin de régler la hauteur de la poutre servant de levier (fig.12). La forme et la profondeur du cuveau rectangulaire où s'exerçait le pressurage paraissent indiquer qu'il servait aussi de fouloir: on aurait donc là un appareil vinaire.

A Fonte do Milho, un massif arasé situé au nord du pressoir devait servir de massif d'ancrage; en revanche, le système de manoeuvre est inconnu. Ailleurs, on en est réduit aux interrogations: en l'absence de bloc d'ancrage de jumelles, on se résoud, faute de mieux, à supposer un ancrage dans une niche murale, tant à São Cucufate, à Torre de Palma, qu'à Monte do Meio (fig. 13).

\subsection{Au Bas-Empire}

Les $\mathrm{IV}^{\mathrm{e}}$ et $\mathrm{V}^{\mathrm{e}}$ siècles de notre ère sont marqués par un extraordinaire essor de la vie rurale. La Péninsule Ibérique se couvre de villae très vastes, luxueuses, décorées de mosaïques, centres de vastes domaines agricoles. Toutes ces exploitations cultivaient des céréales, la plupart s'adonnaient à l'élevage ovin, porcin et bovin et certaines à l'élevage des chevaux (notamment la villa de Torre de Palma). Une grande majorité d'entre elles possédaient également des olivettes et des vignobles, mais notre appréciation de l'ampleur de ces cultures est obérée par le manque de données fiables. La plupart de sites de villae n'ont pas encore livré de témoignage de ces productions et lorsqu'on a trouvé des blocs caractéristiques comme les contrepoids des pressoirs, 
ils sont le plus souvent mal datés et mal décrits dans les publications anciennes, comme à Fontão (Lavra), à Vale de Prazeres, Torre dos Namorados (Castelo Branco), à Beira, Tapada do Garriancho (Portalegre), à Meada, à Tapada Grande, à Alvega, Vale de Cano, Nossa Senhora de Tourega (Évora), à Cardigos, A Moradeira ou à Mexilhoeira Grande, Abicada, à Monte do Poço Seco (Faro)(19). Parfois, la forme des contrepoids est connue: elle est cylindrique à Monte do Meio (Beja: Viana 1957, p. 37), à Pisões (Beja: Ribeiro 1972, pi. 2 montre trois contrepoids de vis cylindriques) ou à Orzalão (Torrão, Setúbal: Faria \& Ferreira 1986, p. 51).

Un pressoir à vis de cette époque a été mis au jour dans la villa de Monte do Meio (Viana 1957, p. 37-40). Il était installé dans une ancienne salle d'apparat décorée d'une mosaïque et doit être rapporté au plus tôt à la fin du IVe siècle. Un petit bassin bétonné en forme de fond de dolium est placé entre la salle de presse et le contrepoids cylindrique du pressoir: il pourrait s'agir d'une cuve de recueil d'huile, mais elle peut aussi bien n'avoir aucun rapport avec l'installation (fig. 13). Les vestiges ne sont pas assez bien décrits et le plan trop incomplet pour que l'on puisse proposer une interprétation assurée. C'est aussi le cas de la villa de Santa Vitória do Ameixial: un ensemble de trois pièces a été interprété comme une installation de pressurage à huile par Chaves (1956, p. 73-75). Elles comportaient des sols en béton de tuileau et la pièce médiane (D) possédait une cuve $(2,76 \mathrm{~m}$ de long, $0,96 \mathrm{~m}$ de large et $0,21 \mathrm{~m}$ de profondeur). La pièce $\mathrm{E}$, profonde de $0,70 \mathrm{~m}$, comportait une cupule d'un diamètre de $0,40 \mathrm{~m}$, dont Chaves fait un support dans lequel tournait la vis du pressoir. Là encore la description et le plan (fig. 14) sont insuffisants pour se prononcer. Outre le fait que ces constructions ne sont pas précisément datées, elles ne présentent aucune disposition permettant de les interpréter comme des aires de presse. Il est possible qu'il s'agisse en fait de cuves partiellement arasées: l'espace E serait alors classiquement pourvu d'une cupule de nettoyage et la faible profondeur de la cuve $\mathrm{Db}$ trouverait une explication. Dans cette hypothèse, il faudrait restituer des pressoirs à l'est entre le mur A et le mur $\mathrm{H}$. Leur sol ayant été totalement détruit, n'en subsisteraient que les fondations. Aller plus loin, notamment à propos de la détermination du produit traité, serait hasardeux.

(19) Pour tous ces sites, je renvoie à la somme de J. de Alarcâo, Roman Portugal, Gazeteer 1988, sub nomine.

Conimbriga, 36 (1997) 45-72 
Dans quelques cas privilégiés, on peut préciser la destination des installations comme à Estoi (Milreu), à Torre de Palma (Monforte) et à São Cucufate (Vila de Frades). Dans la villa d'Estoi à Milreu (Hanel 1989), l'installation viticole comprend un fouloir, un pressoir et des cuves de recueil, mais on ne connaît pas le mode de vinification.

A Torre de Palma, les vestiges agricoles situés à l'ouest de la grande cour précédant la demeure aristocratique du Bas-Empire, comprennent un pressoir, deux ou peut-être trois cuves et un vaste entrepôt que Maloney \& Hale (1996) attribuent à leur phase IV, datée du troisième quart du IVe siècle. Qualifiée d'huilerie à la suite des premières fouilles de M. Heleno, cette installation doit vraisemblablement être attribuée au vin contrairement à ce que proposent Oliveira (1967) et Maloney \& Hale (1996, p. 292). Il faut tenter de le démontrer:

- l'installation ne comporte pas de moulin, ni de meule;

- la plateforme du pressoir est vaste; il y aurait la place pour installer deux pressoirs à leviers, mais il n'y a qu'un contrepoids;

— un fond de petite jarre est inséré dans le sol du pressoir (Oliveira 1967, fig. 2): son emplacement pourrait faire penser que l'on a affaire à un pressoir à huile: un tel aménagement se conçoit lorsqu'on a besoin de récupérer l'huile de première pression avant d'envoyer l'huile et l'eau chaude de seconde pression dans des cuves plus grandes où s'effectuera la décantation. Toutefois cet indice n'est pas dirimant, car certains pressoirs à vin des environs de Pompéi sont équipés de petits dolia bâtis dans leur pavement et connectés avec le sol de la presse par un canal (villa della Pisanella par exemple: Pasqui 1897, p. 468);

- les cuves de recueil sont grandes, mais ce n'est pas non plus un critère de discrimination (Brun 1993B);

- elles sont construites dans un vaste entrepôt mesurant environ $43 \mathrm{~m}$ de longueur par $14 \mathrm{~m}$ de largeur et rythmé par des piliers qui délimitent une allée centrale et vingt compartiments (fíg. 16). Cet entrepôt a été ultérieurement cloisonné et transformé en pièces d'habitation (phase V: V-VIP siècles), mais il est évident que, au cours de la phase productive, il était libre de tout aménagement bâti.

Ce type de grand hangar, rythmé par des piliers et comportant des cuves, est connu en Italie (villa de Russi: Corlaita Scagliarmi 1975) et en Narbonnaise (villa de Saint-Barthélémy de Montgey, Tarn: Passelac 1986). Ils semblent caractéristiques d'installations viticoles où l'on 
vinifiait non dans des jarres de terre-cuite, mais dans des vaisseaux de bois. La villa de Russi est située dans la région de Ravenne, dans cette Cisalpine de peuplement gaulois où, selon Strabon, la vinification était conduite dans de grands foudres.

On peut interpréter ces observations dans le cadre d'une production de vin d'autant plus que la culture de la vigne est attestée par la découverte de serpettes de vignerons (Alves 1989, p. 94). Grâce à une ouverture pratiquée dans le mur nord du pressoir, le raisin était déversé dans la partie nord de la plateforme qui servait de fouloir. Le moût pouvait s'écouler dans l'une ou l'autre des cuves. Lorsque le raisin était assez foulé, on le pressurait immédiatement et le jus s'écoulait dans une des cuves. L'intérêt de posséder plusieurs cuves de recueil du moût résidait dans la possibilité de vider l'une pendant que l'on utilisait l'autre et éventuellement de séparer les jus issus du foulage de ceux extraits à la presse. On sait que les jus de presse n'ont pas la même composition que les moûts tirés du foulage et que Pline recommandait notamment de ne pas mêler aux autres le jus provenant du marc de deuxième serre $\left({ }^{20}\right)$. Le moût était ensuite transvasé des cuves dans des récipients pour que la fermentation puisse s'effectuer. Le grand hangar était susceptible de loger vingt foudres. En laissant un espace de circulation autour des piliers, on pourrait restituer des foudres mesurant $1,80 \mathrm{~m}$ de diamètre et $3,40 \mathrm{~m}$ de longueur, soit d'une capacité moyenne de $85 \mathrm{hl}$, ce qui donnerait une capacité théorique de production maximale de $1700 \mathrm{hl}$. Pour relativiser ce chiffre, il faut le comparer à ceux obtenus dans les domaines viticoles d'Italie et de Narbonnaise (voir, en dernier lieu, Tchernia 1995). Les villae italiennes ont des capacités de vinification variant de $100 \mathrm{hl}$ (villa Regina) à $792 \mathrm{hl}$ \{villa de la Pisanella) et à $880 / 1100 \mathrm{hl}$ \{villa de Settefinestre). Il faut aller dans la province de Narbonnaise pour dépasser ces chiffres: la villa de Cavalaire (Var) présentait des capacités de production pouvant atteindre $1700 \mathrm{hl}$, celle de La Roquebrussanne (Var) $1020 \mathrm{hl}$, celle de Donzères (Drôme) 2500/ $13000 \mathrm{hl}$ et celle de Rians (Var) au moins autant (Brun \& Congés 1994). A. Tchernia retient une fourchette de rendement comprise entre $35 \mathrm{hl}$ et $60 \mathrm{hl}$ à l'hectare au maximum. Les plus grands de ces domaines

${ }^{(20)} \quad$ Vairon $R$. R. I, 54, 3: “Lorsque le marc sous le pressoir a cessé de s'écouler, certains coupent tout ce qui dépasse et le pressent à nouveau et après ce nouveau pressurage obtiennent ce qu'on appelle le vin de rognure et le conservent à part car il a un goût de fer".

Conimbriga, 36 (1997) 45-72 
pouvaient donc posséder entre 40 et 80 hectares de vignes. Si l'on applique les mêmes ratios à Torre de Palma, il faudrait estimer que le vignoble couvrait entre 30 et 50 hectares.

A São Cucufate, les fouilleurs n'ont décelé aucun changement dans la zone des pressoirs au cours du Bas-Empire. Plus au sud, toutefois, un vaste entrepôt fut alors construit. Il s'agit d'un bâtiment large de $12 \mathrm{~m}$ et dont la longueur excède $20 \mathrm{~m}$; l'évolution de son plan n'est pas claire, mais il semble bien que, primitivement, il ait été divisé en deux nefs par une série de piliers axiaux. Là encore, il pourrait s'agir d'un vaste chai à vin abritant des foudres qui, dans un second temps aurait été cloisonné et transformé en pièces à usages indéterminées.

Comme pour le Haut-Empire, la production d'huile reste mal connue (on ne peut que lui attribuer, sous toutes réserves, que l'installation de Monte do Meio). Cette anomalie tient certainement au manque de prospection systématique et à la rareté des dégagements des parties agricoles. En effet, les vestiges les plus caractéristiques de la production de $\mathrm{P}$ huile, les meules et les moulins, ont tendance à disparaître plus aisément que ceux des pressoirs, plus encombrants et peu réutilisables (Brun 1993B). Gageons que l'intérêt suscité par les productions agricoles et la vie économique amènera sous peu une documentation renouvelée sur ce sujet.

Il reste qu'il faut se poser la question de l'existence de régions de production spécialisée et celle des débouchés commerciaux. L'état actuel de la documentation privilégie 1'Alentejo comme grande région viticole dès le Haut-Empire, mais il existe quelques sites de production dans la vallée du Douro et il est possible que cette région soit sous représentée dans la documentation. Pour l'huile, on manque encore de données pour se prononcer. Quant aux débouchés, une vaste enquête reste à mener d'une part pour tenter de trouver les lieux de consommation des amphores Lusitaniennes 3 qui ont dû souvent être confondues dans les publications avec des amphores gauloises 4 ou Dressel 30. Si notre hypothèse de vinification en foudres est exacte, il en découle que la majorité du vin produit à partir du $\mathrm{II}^{\mathrm{e}}$ siècle dut être commercialisé en tonneaux. On buterait alors sur une des limites des sources archéologiques, la conservation des documents; à moins de découvrir de nouvelles sources écrites, la question de la diffusion du vin lusitanien depuis les Antonins jusqu'au Bas-Empire a toutes chances de rester insoluble. 


\section{BIBLIOGRAPHIE}

AKerraz (A.), LenOIR (M.) - Les huileries de Volubilis, Bulletin d'archéologie marocaine, XIV, 1981-1982, p. 69-120.

AlarCão (J. de) - A vida rural no Alentejo na época romana. Coimbra, 1974.

Alarcão (J. de), Etienne (R.) — Fouilles de Conimbriga. I. L'architecture. Paris, De Boccard, 1977.

AlarCão (J.) - Les amphores, in: Alarcão (J.), Etienne (R.) — Fouilles de Conimbriga VI. Paris, De Boccard, 1977, p. 79-91.

Alarcão (J. de) - Portugal romano. Lisboa, Verbo, 1983.

Alarcão (J. de) - Roman Portugal, Warminster, Aris \& Phillips, 1988. (vol I, Introduction; vol II, Gazetteer: 1, Porto, Bragança \& Viseu; 2, Coimbra \& Lisboa; 3, Évora, Faro \& Lagos).

Alarcão (J. de), Etienne ( R.), Mayet (F.) (dir.) - Les villas romaines de São Cucufate (Portugal). Paris, De Boccard, 1990.

Alfenim (R.), Lopes (C.) — La villa romaine de Monte da Cegonha, Les dossiers d'archéologie n. ${ }^{\circ}$ 198, Le Portugal, Novembre 1994, p. 64-67.

Alves (Fr.) (dir.) — Le Portugal, des origines à l'époque romaine. Lisboa, Museu Nacional de Arqueologia e Etnologia, 1989.

BRUn (J.-?.) - L'oléiculture antique en Provence, Les huileries du département du Var. $15^{\mathrm{ème}}$ Supplément à la Revue archéologique de Narbonnaise. Paris, CNRS, 1986.

BRUN (J.-P.) - Les innovations techniques et leur diffusion dans les pressoirs, in: Amouretti (M.-C.) \& Brun (J.-P.) (éds.) - La production du vin et de l'huile en Méditerranée. Supplément au B. C. H. Athènes, 1993A, p. 539-550.

BRUN (J.-P.) - La discrimination entre les installations oléicoles et vinicoles, in: Amouretti (M.-C.) \& Brun (J.-P.) (éds.) - La production du vin et de l'huile en Méditerranée. Supplément au B. C. H. Athènes, 1993B, p. 511-537.

BRUN (J.-P.) \& CONGÉs (G.) - La villa viticole romaine des Toulons (Rians, Var), Annales de la Société des sciences naturelles et d'archéologie de Toulon et du Var, 1994, p. 219-241.

CArdoso (G.) - O forno de ânforas de Muge, in: Alarcão (A.), Mayet (F.) - As ânforas lusitanas /Les amphores lusitaniennes. Conimbriga, Museu, 1990, p. 153$-166$.

CARdoso (G.), Encarnação (J. d') - A villa romana de Freiria e o seu enquadramento rural, Studia Historica, Historia Antigua (Salamanca), X-XI, 1992-1993, p. 203$-217$.

CARdoso (G.), Encarnação (J. d') - 10. a campanha na villa romana de Freiria (Cascais), Al-madan, 4, 1995, p. 167.

Chaves (L.) - Estudos lusitano-romanos I: A villa luso-romana de Santa Vitoria do Ameixial (Concelho de Estremoz), O Arqueólogo Português, XXX, 1956, p. 14-117.

Corlaita Scagliarini (D.) - Russi, la villa romana, la città. Faenza, Società di Studi Romagnoli, 1975.

Diogo (A. M. D.) - Quadro tipològico das ânforas de fabrico lusitano, O Arqueólogo Português, IV, 1987, p. 179-191. 
Diogo (A. M. D.), Faria (J. C. L.) - Fornos de cerâmica romana no vale do Sado: alguns elementos, in: Alarcão (A.), Mayet (F.) - As ânforas lusitanas/Les amphores lusitaniennes. Conimbriga, Museu, 1990, p. 173-186.

Diogo (A. M. D.), Alves (F.) - Ânforas lusitano-romanas provenientes do meio fluvial, O Arqueólogo Português, IV, 6/7, 1988-1989, p. 227-240.

Encarnação (J. d') - Inscrições romanas do Conventus Pacensis. Coimbra, Instituto de Arqueologia da Faculdade de Letras, 1984.

FABĨ̃o (C.) —Sobre a tipologia das ânforas da Lusitânia, in: Filipe (Gr.), Raposo (J. M. C.) - Ocupação romana dos estuários do Tejo e do Sado. Lisboa, Dom Quixote, 1996, p. 371-390.

FABião (C.), CARvalho (A.) - Ânforas da Lusitânia: uma perspectiva, in: Alarcão (A.), Mayet (F.) - As ânforas lusitanas/Les amphores lusitaniennes. Conimbriga, Museu, 1990, p. 37-63.

FABIÃo (C.) - O azeite da Bética na Lusitânia, Conimbriga, XXXII-XXXIII, 1993-1994 , p. 219-245.

Freijeiro (A. B.) - Mosaicos romanos de Merida, Corpus de mosaicos romanos de España, fase 1. Madrid, 1978.

GonZÁlez Fernández ( J. ) - Nueva inscripción de un diffusor olearius en la Betica, in: Producción y comercio del aceite en la Antigüedad, Segundo congresso internacional, Sevilla, 1982. Madrid: Universidad Complutense, 1983, p. 183-191.

GONZÁLez Fernández (J.) - Corpus de inscripciones latinas de Andalucía, 2, Sevilla, 1991.

Gorges (J.-G.) - Les villas hispano-romaines: Inventaire et problématique archéologiques. Paris, De Boccard, 1979.

Gorges (J.-G.) - Villas romaines de Lusitanie, Bulletin des Études Portugaises et Brésiliennes, 46-47, 1987, p. 149-170.

Gorges (J.-G.) - Villes et villas de Lusitanie: Intéractions, échanges, autonomies, in: Les villes de Lusitanie romaine. Paris, CNRS, 1990, p. 91-113.

HANEL (N.) - Römische Öl- und Weinproduktion auf der Iberischen Halbinsel am Beispiel von Munigua und Milreu, Madrider Mitteilungen, 30, 1989, p. 204-238.

Julia (D.) - Les monuments funéraires en forme de demi-cylindres en Tarraconnaise, Mélanges de la Casa de Vélasquez, I, 1965, p. 29-54.

LEENWARden (W. V.), JANSSEN (C. R.) - A preliminary palynological study of peat deposits near an oppidum in the Lower Tagus Valley, Portugal, Actas da Reunião do Quaternário Ibérico, II, 1985, p. 225-236.

Leveau (P.), Heinz (C.), Laval (H.), Marinval (P.), Medus (J.) - Les origines de l'oléiculture en Gaule du Sud. Données historiques, archéologiques et botaniques, Revue dArchéométrie, 15, 1991, p. 83-94.

Leveau (Ph.), Sillières (P.), Vallat (J.-P.) - Campagnes de la Méditerranée romaine. Paris, Hachette, 1993.

Lima (J. F.) - Aspectos da romanização no território português de Bética, $O$ Arqueólogo Português, 2, 1, 1951, p. 171-211.

Maloney (St. J.), Hale (J. R.) - The villa of Torre de Palma (Alto Alentejo), Journal of Roman Archaeology, 9, 1996, p. 275-294. 
Mantas (V. G.) - As cidades marítimas da Lusitânia, in: Les villes de Lusitanie romaine. Paris, CNRS, 1990, p. 149-205.

Matos (J. L. de) (dir.) - Inventário do Museu Nacional de Arqueologia. Colecção de Escultura Romana. Lisboa, 1995.

MAYET (F.) - Typologie et chronologie des amphores lusitaniennes, in: Alarcão (A.), Mayet (F.) - As ânforas lusitanas/Les amphores lusitaniennes. Conimbriga, Museu, 1990, p. 29-35.

Mayet (F.), Tavares da Silva (e.) — L'établissement phénicien d'Abul (Alcácer do Sal), Les dossiers d'archéologie n. ${ }^{\circ} 198$, Le Portugal, Novembre 1994 (A), p. 22-23.

Mayet (F.), Tavares da Silva (e.) - Les industries de salaisons de poisson dans la basse vallée du Sado, Les dossiers d'archéologie n. ${ }^{\circ} 198$, Le Portugal, Novembre 1994 (B), p. 68-73.

Mayet (F.), Schmitt (A.), Tavares da Silva (e.) - Les amphores du Sado, Portugal. Paris, De Boccard, 1996.

MesQuiriz Irujo (M.-A.) - La villa de las musas (Arellano-Navarra). Estudio previo. Trabajos de Arqueologia Navarra, 11, 1993, p. 55-90.

Oliveira (A. J. S.) - O lagar romano de Palma, Boletim da Junta de Provinda de Alto Alentejo, 1958, p. 5-13.

Oliveira (A. J. S.) - A villa lusitano-romana de Torre de Palma (Monforte), Lavoura Portuguesa, 3-4, Abril 1967, p. 10-18.

Panciera (S.) - Olearii, in: D’Arms (J. H.), Kopff (E. C.) (eds) - The Seaborne Commerce of Ancient Rome, Studies in Archaeology and History. Rome, American Academy, 1980, p. 235-250.

PAsQui (A.) - La villa pompeiana della Pisanella presso Boscoreale, in: Monumenti antichi, VII, Roma, 1897.

Passelac (M.) - Observations aériennes et sondages sur la villa de Saint-Barthelemy à Montgey (Tarn), in: Mélanges Labrousse, 1986, p. 303-321.

Pimenta (Fr. C.) - Subsídios para o estudo do material anfórico conservado no Museu Regional de Sintra, Sintria, I, 1982-1983, p. 117-150.

Pinto (C. V.), PARreira (R.) - Contribuição para o Estudo do Bronze Final e do Ferro a Norte do Estuário do Tejo, Actas das III Jornadas Arqueológicas, 1978, p. 147-163.

Pinto (I. V.), Viegas (C.) — Les thermes de la villa romaine de Tourega, Les dossiers d'archéologie n. ${ }^{\circ} 198$, Le Portugal, Novembre 1994, p. 60-63.

Ponte (M. S.), Guimarães (M.), Pessoa (M.), Marques (A. P.) - La production de l'huile et du vin au Portugal durant l'Antiquité et le Moyen-Age in: Amouretti (M.-C.) \& Brun (J.-P.) (éds.) — La production du vin et de l'huile en Méditerranée. Supplément au B. C. H. Athènes, 1993, p. 413-421.

Raposo (J. M. C.) - Porto dos Cacos: uma oficina de produção des ânforas romanas no vale do Tejo, in: Alarcão (A.), Mayet (F.) — As ânforas lusitanas /Les amphores lusitaniennes. Conimbriga, Museu, 1990, p. 117-151.

Raposo (J. M. C.), Duarte (A. L.) - O forno 2 do Porto dos Cacos (Alcochete), in: Filipe (Gr.), Raposo (J. M. C.) - Ocupação romana dos estuários do Tejo e do Sado. Lisboa, Dom Quixote, 1996, p. 249-266.

Conimbriga, 36 (1997) 45-72 
Ribeiro (F. N.) - A villa luso-romana de Pisões. Beja, 1972.

Russel CoRtez (F.) - As escavações arqueológicas do "castellum" da Fonte do Milho, Anais do Instituto do Vinho do Porto, 12, 1951, p. 17-88.

Santos Rocha (A.) - Antiguidades de Marim, O Archeologo Português, I, 1895, p. 113-116.

Santos Rocha (A.) - Notícia de algumas estações romanas e árabes do Algarve, O Archeologo Português, II, 1896, p. 65-79.

Santos Rocha (A.) - Antiguidades romanas das vizinhanças de Nelas, O Archeologo Português, III, 1897, p. 81-86.

Sciallano (M.), Sibella (P.) - Amphores, comment les identifier? Aix, Edisud, 1991.

Severo (R.) - Notícia da estação romana na Quinta da Ribeira em Tralhariz, Portugalia, I, 2, 1900, p. 391-398.

TChernia (A.) - Amphores et marques d'amphores de Bétique à Pompéi et à Stabies, MEFR, 1964, p. 419-449.

TCherNia ( A.) - Le vin de VItalie romaine: Essai d'histoire économique d'après les amphores. Rome, Ecole française, 1986.

TCHERNIA (A.) - Les dimensions de quelques vignobles romains, in: Du Latifundium au Latifondo. Bordeaux, CNRS, 1995, p. 383-394.

Teixeira (C.) - Estação romana de Canelas (Poiares da Régua), Trab, da Soc. Port, de Ant. e Etnol, IX, 1940, p. 130-135.

Viana (A.) - Museu Regional de Beja, Arqueologia de Beja, II, Secção lapidar, Arquivo de Beja, II, 1945, p. 97-128 et 321-322.

Viana (A.) - Ruínas romanas do Monte do Meio, Arquivo de Beja, XI, 1954, p. 13-16.

Viana (A.) - Villa romana do Monte do Meio, Arquivo de Beja, XVI, 1959, p. 36-43.

Viana (A.), Formosinho (J.), Ferreira (O. Veiga) - De lo prerromano a lo árabe en el museo regional de Lagos, AEA, 1953, p. 128-130.

Whittaker (C. R.) - Les frontières de l'Empire romain. Paris, Les Belles Lettres, 1989.

Conimbriga, 36 (1997) 45-72 


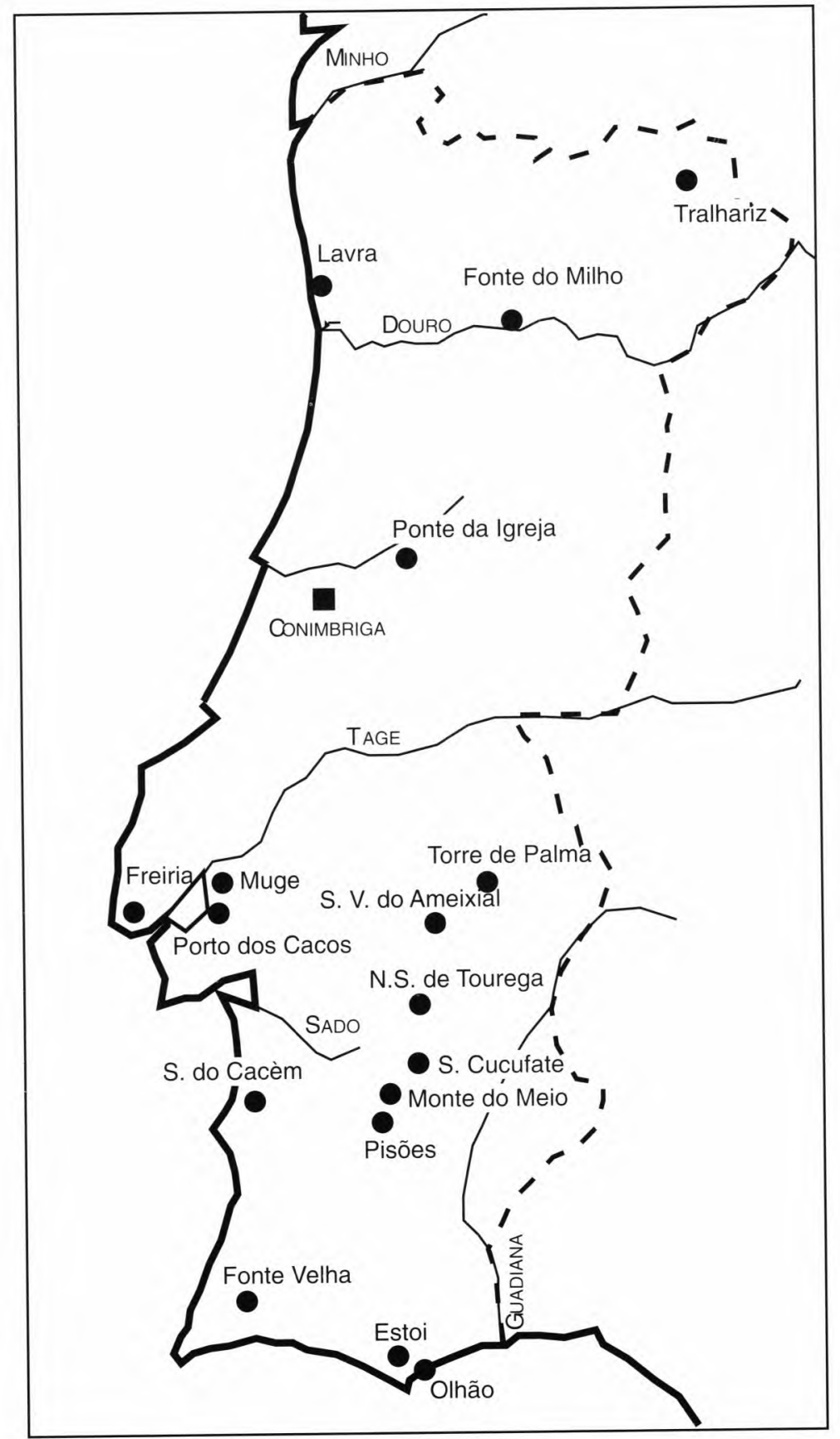

FIG. 1 - Carte des sites mentionnés. 


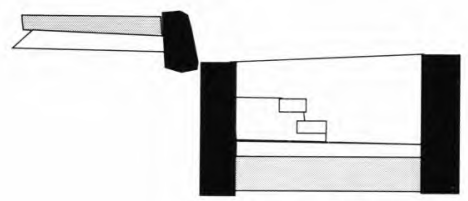

Coupe A-B

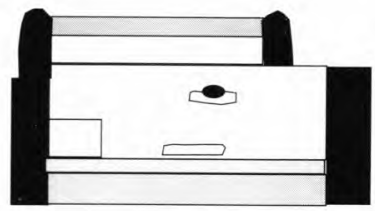

Fouloir

Coupe C-D

0

$1 \mathrm{~m}$

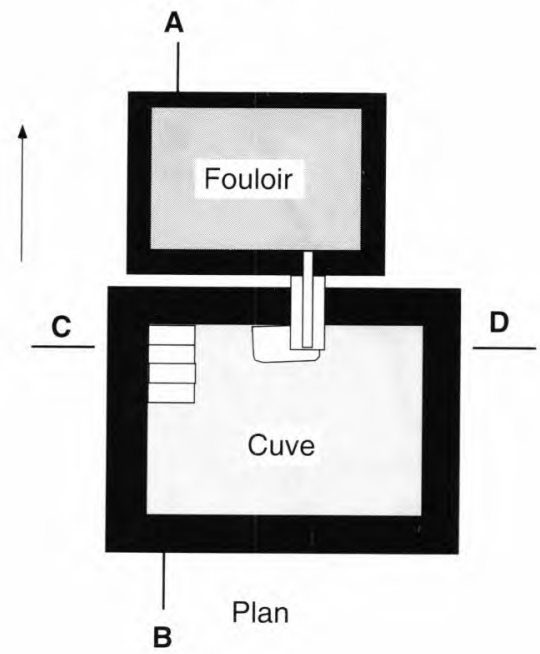

FIG. 2 - Fouloir de Ponte da Igreja, d'après Russell Cortez 1951, fig. 24. 


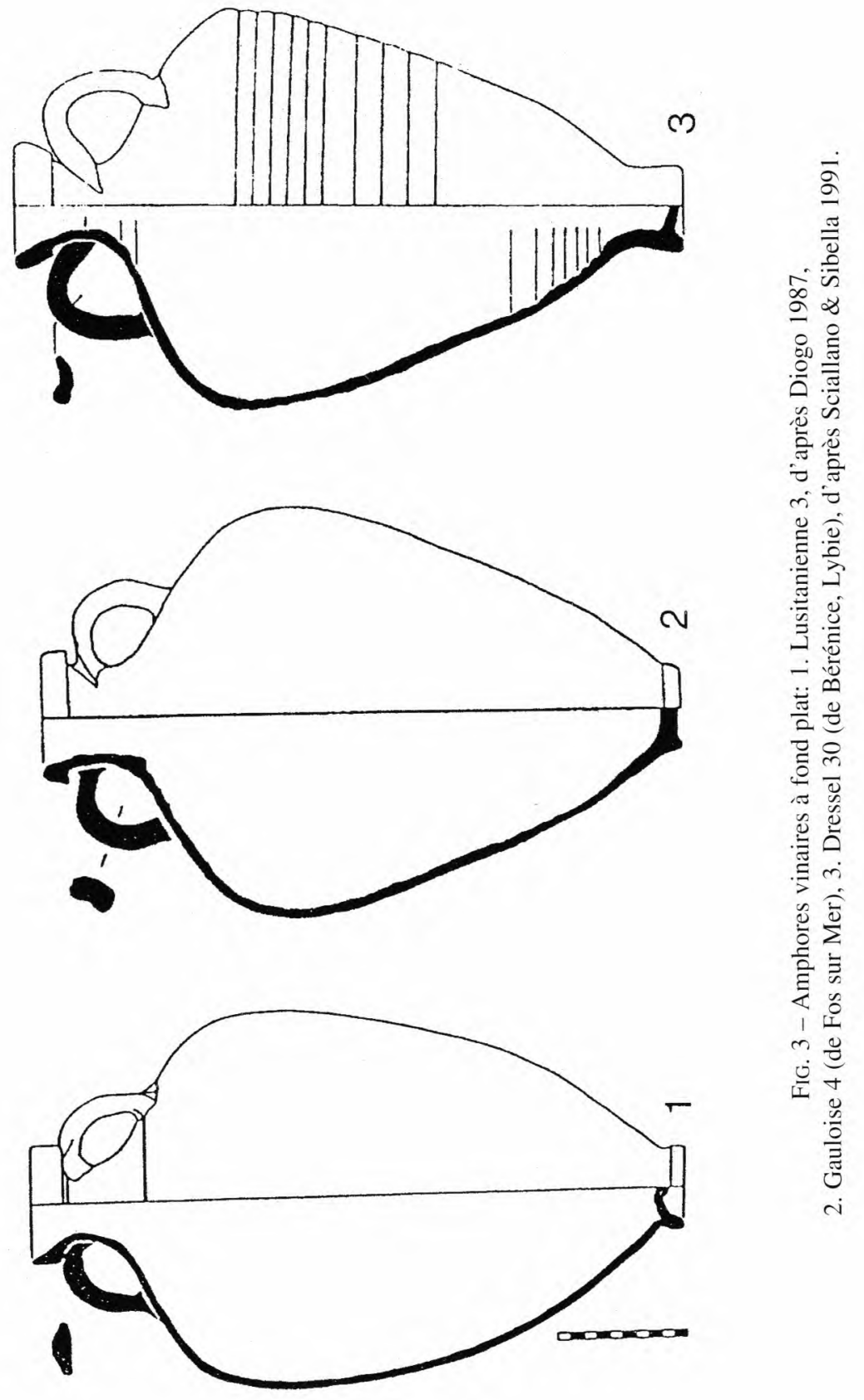




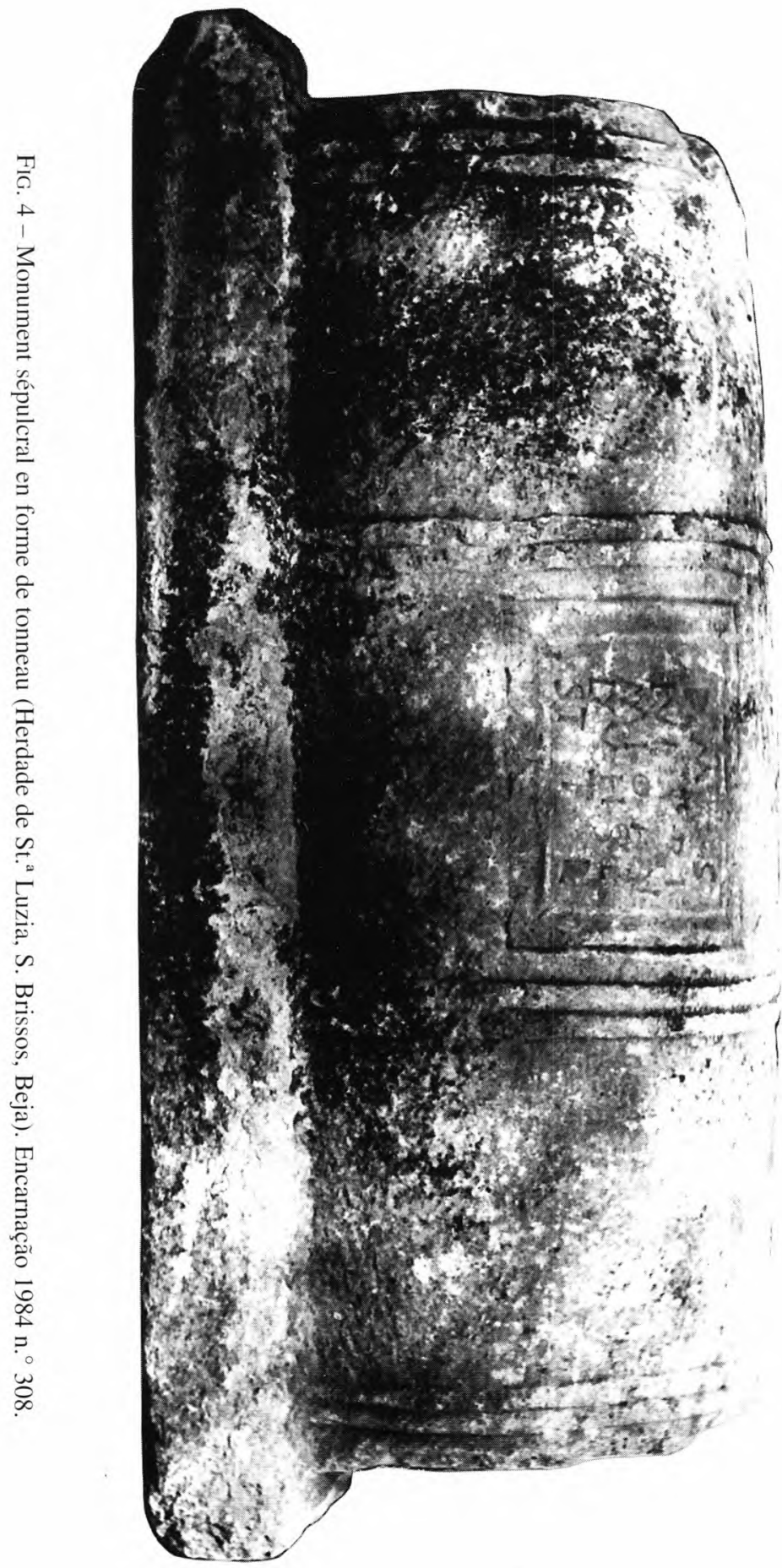




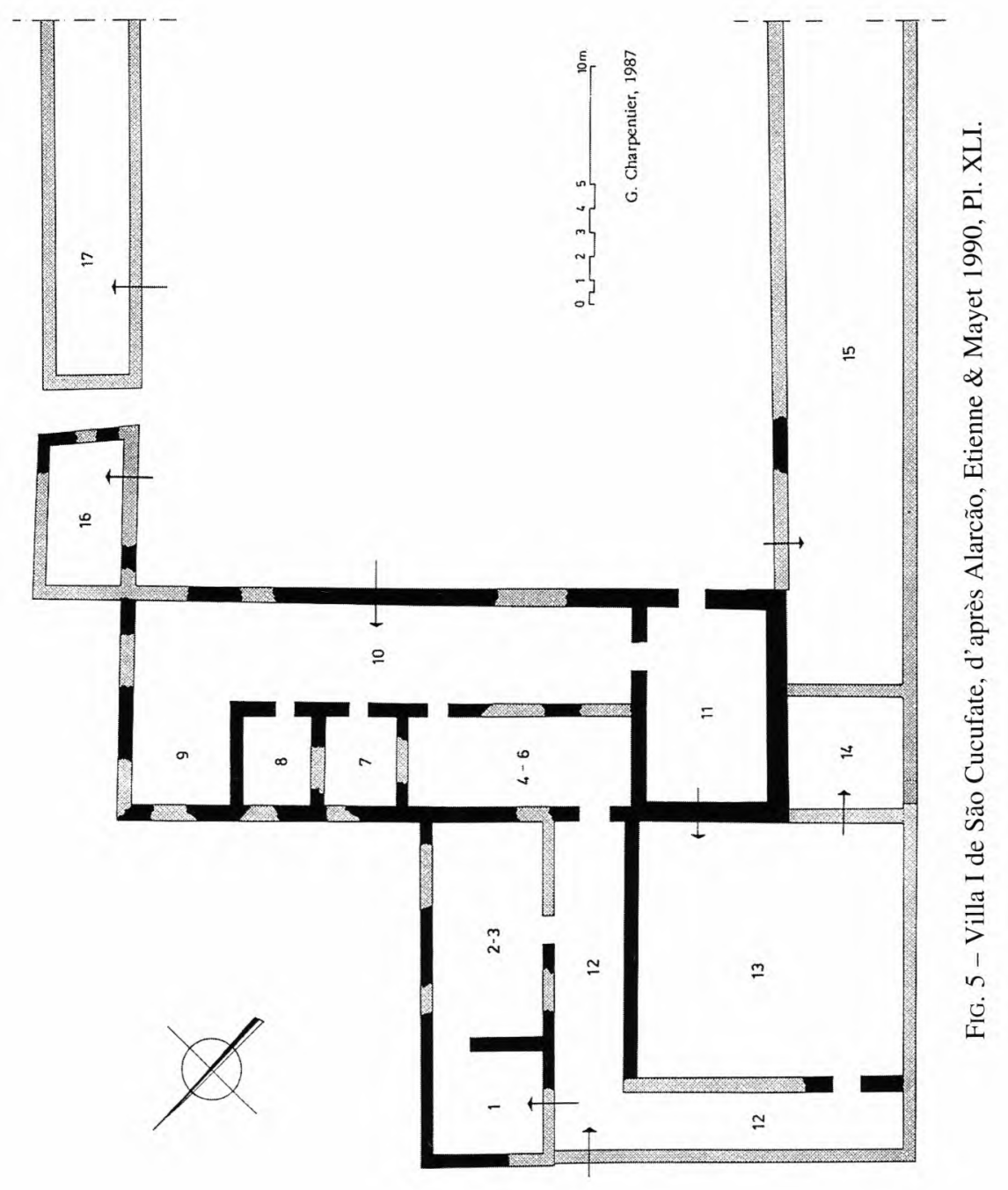




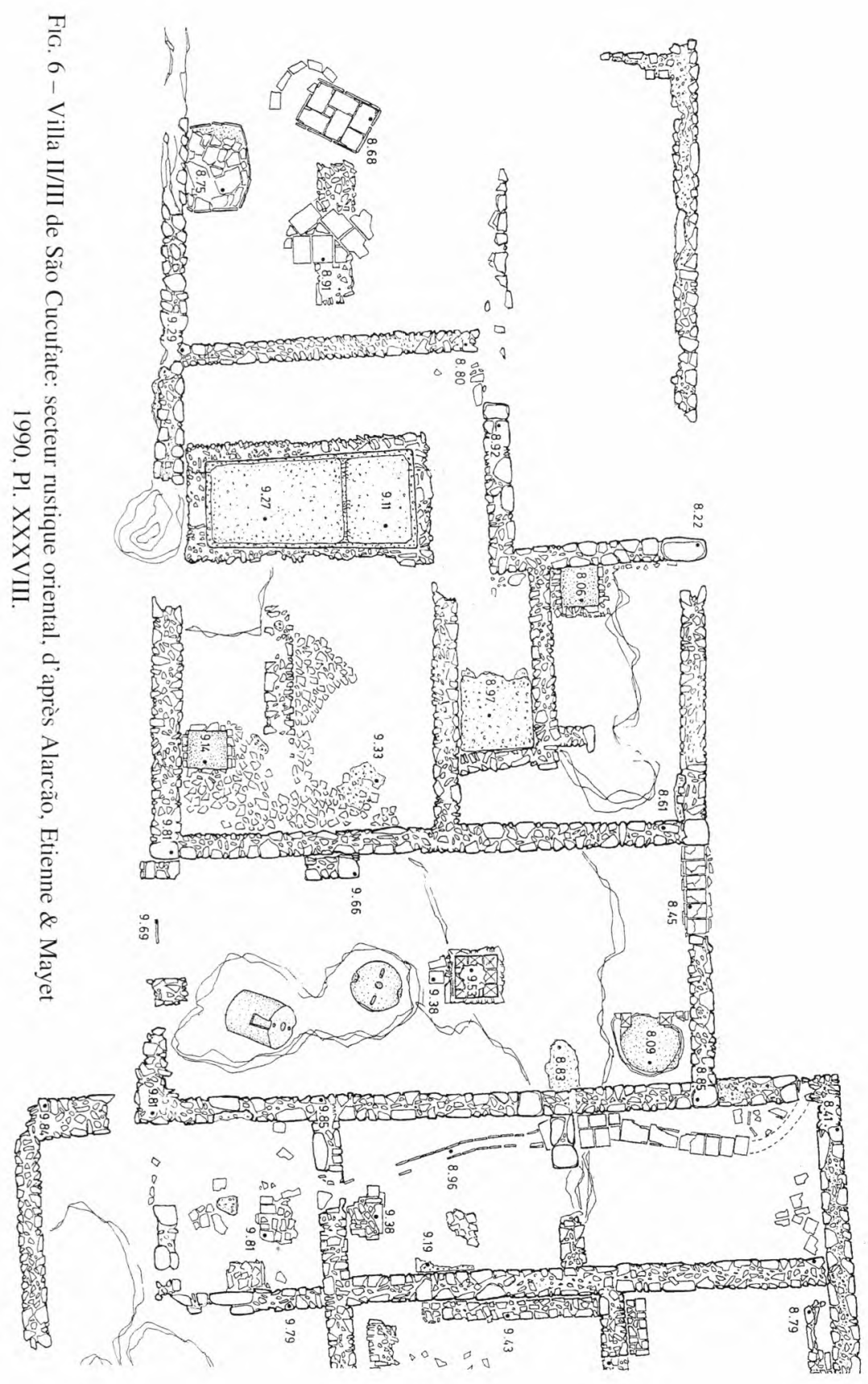




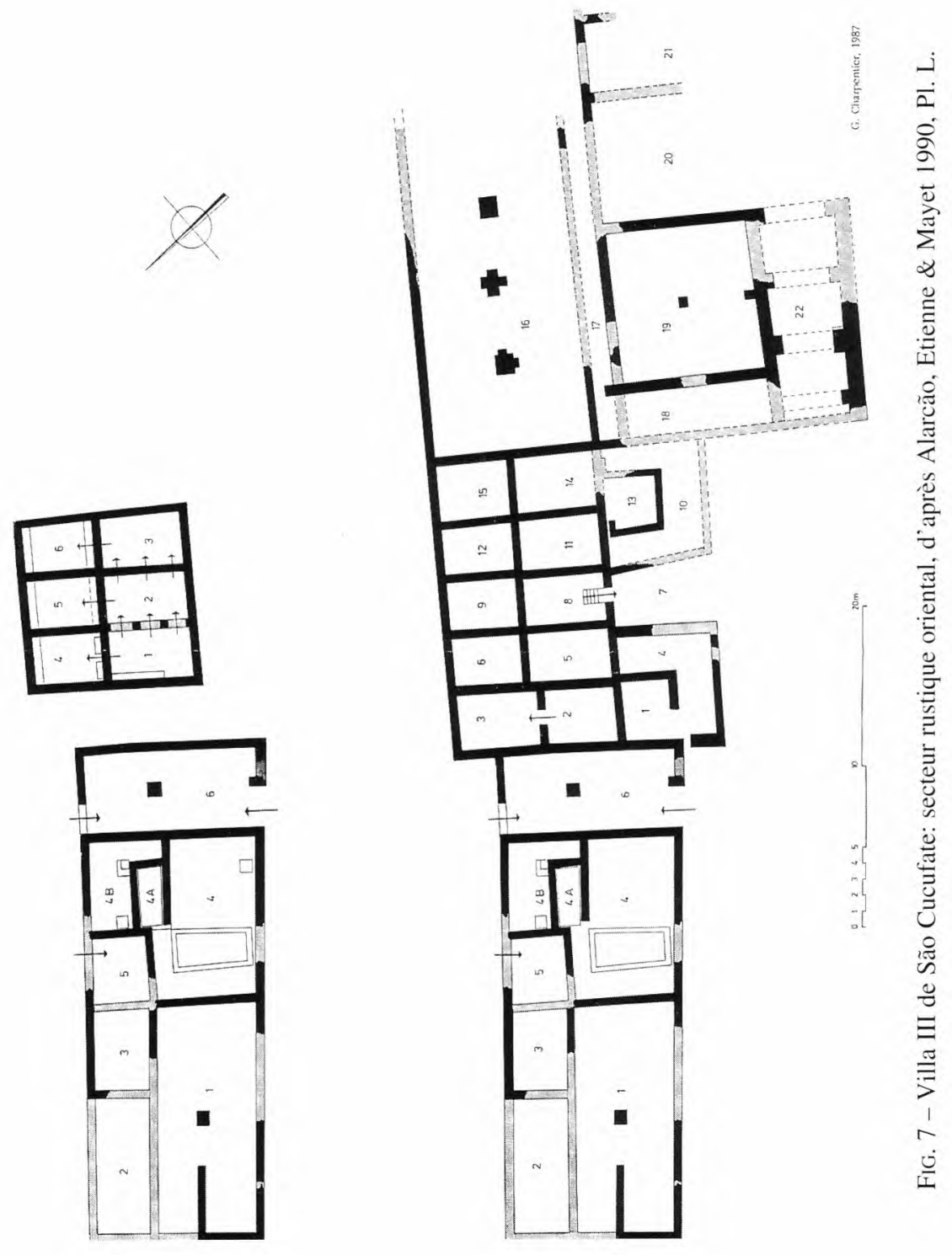




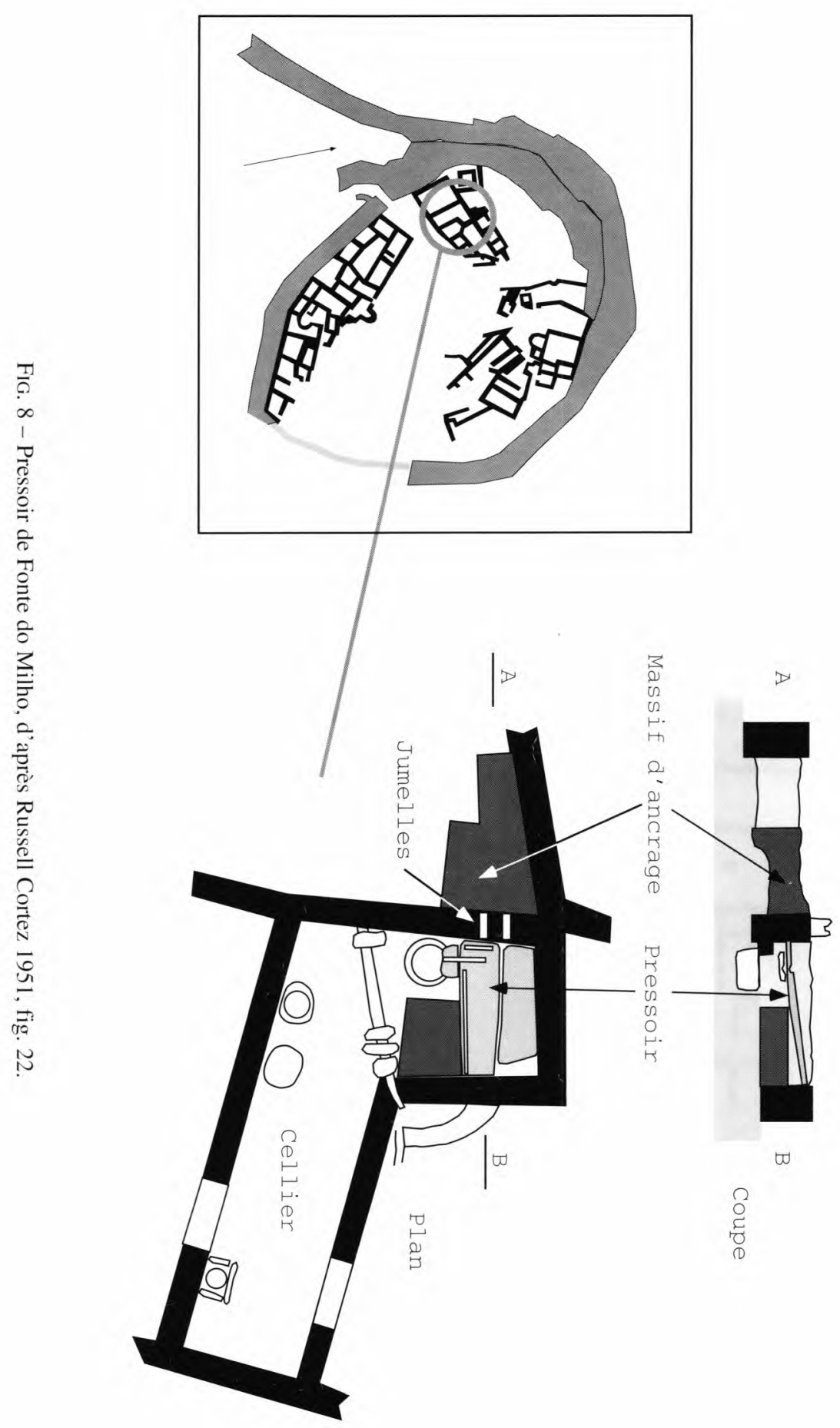




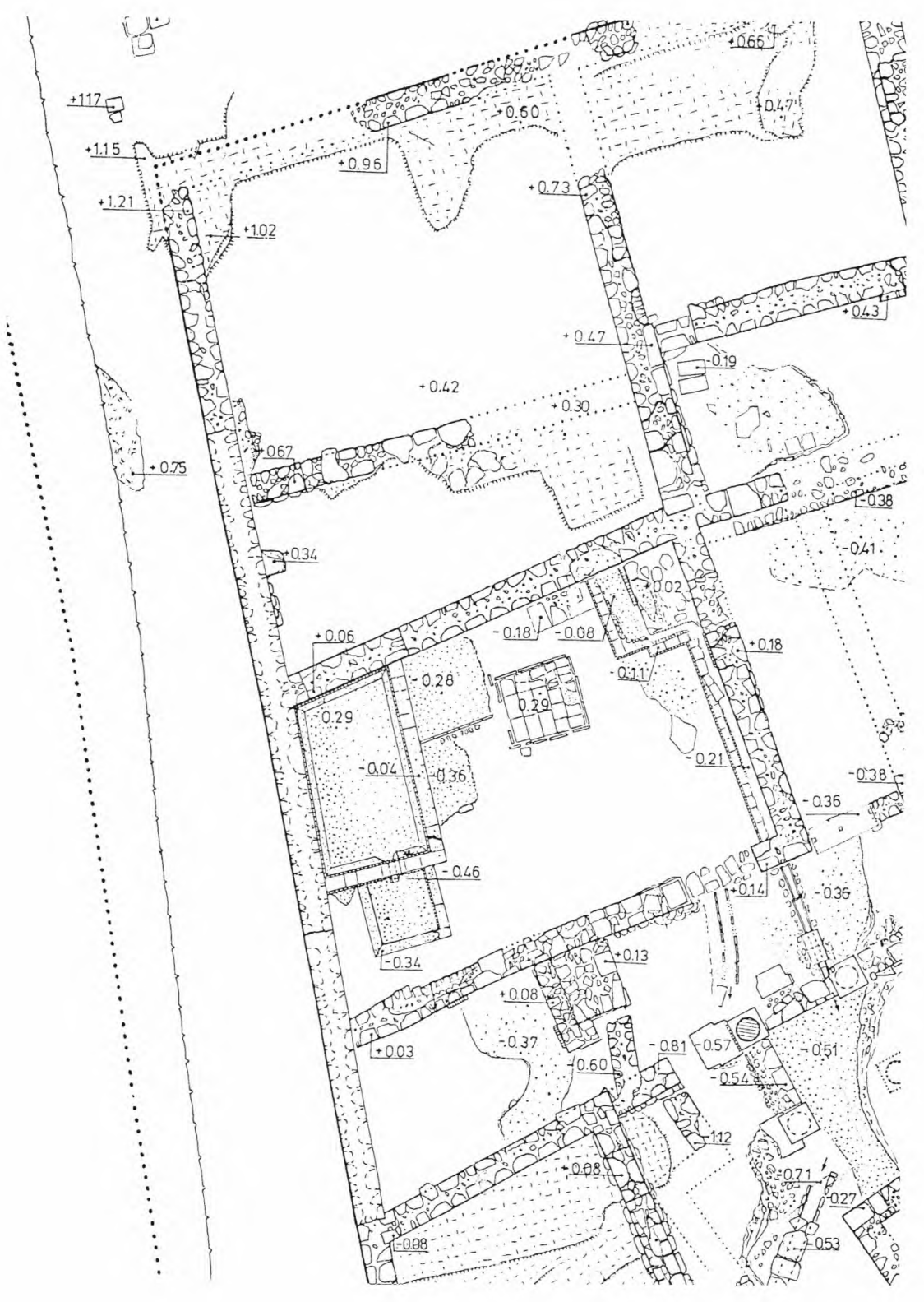

FIG. 9 - Fouloir à vin de Conimbriga, d'après Alarcão et Etienne, 1977, Pl. XXXIX. 


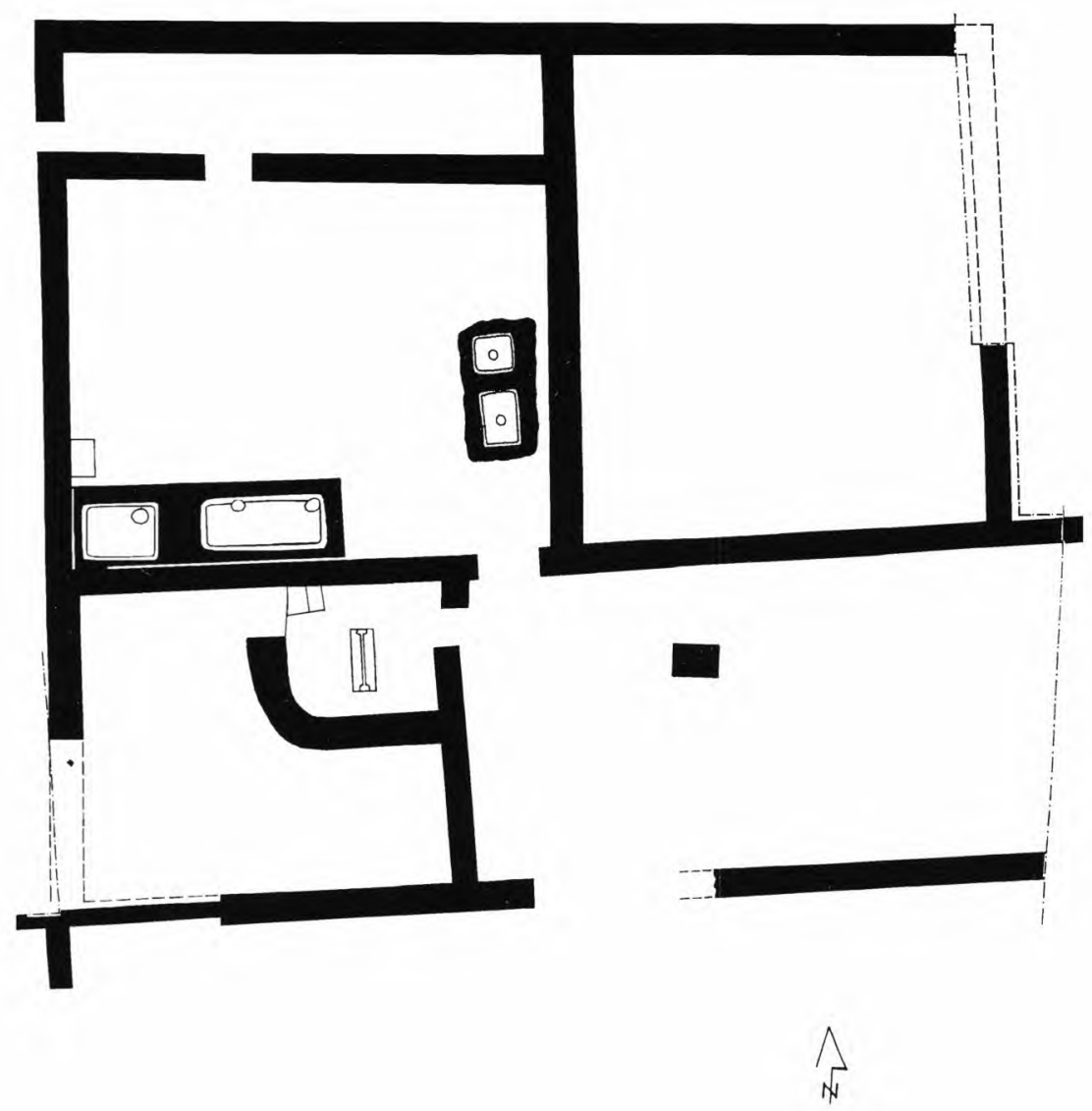

Fig. 10 - Pressoir de la villa de Freiria, plan d'après Cardoso \& Encarnação. Esc. 1.200 


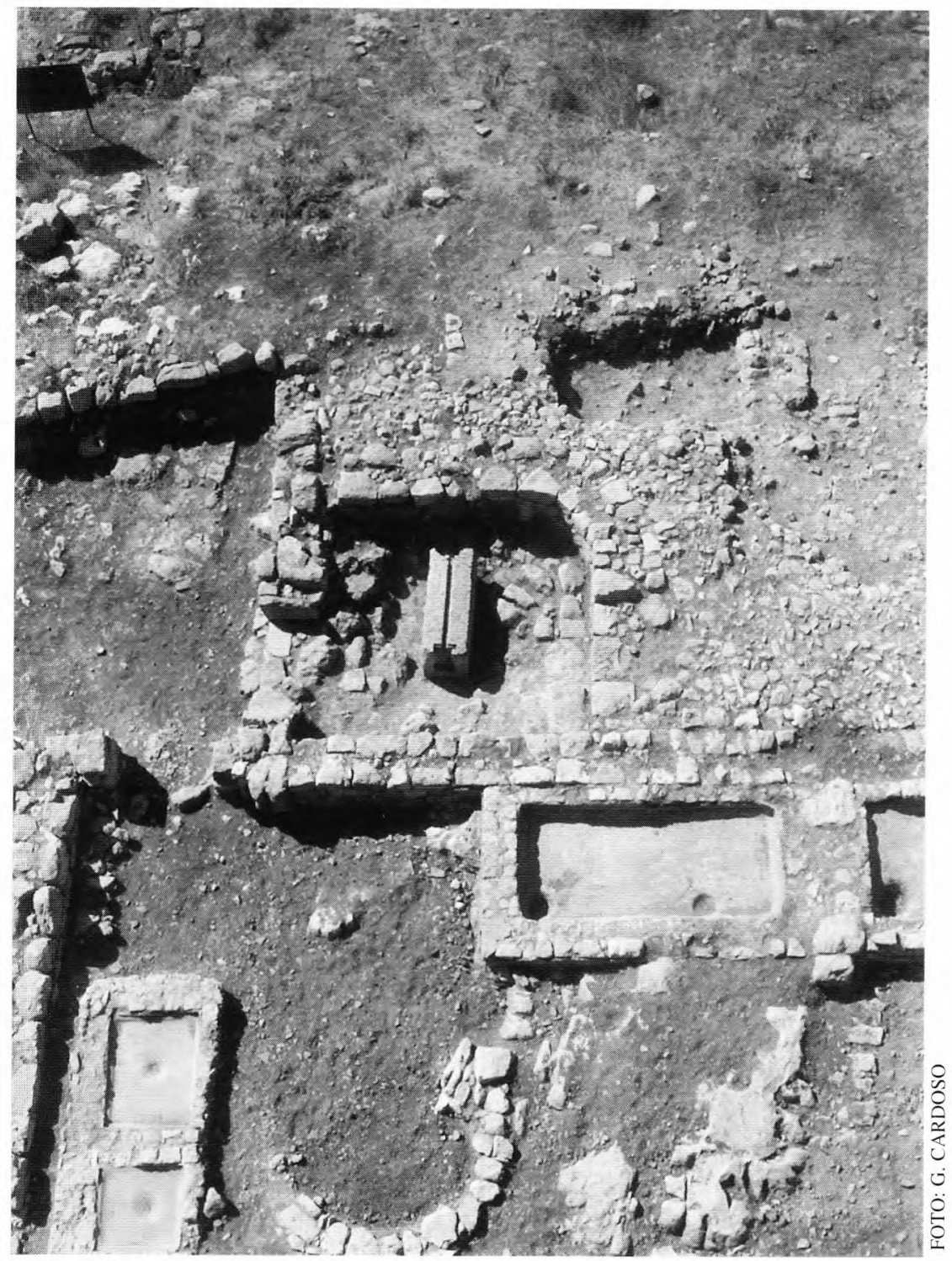

FIG. 11 - Pressoir de la villa de Freiria, photographie aérienne, d'après Cardoso \& Encarnação. 


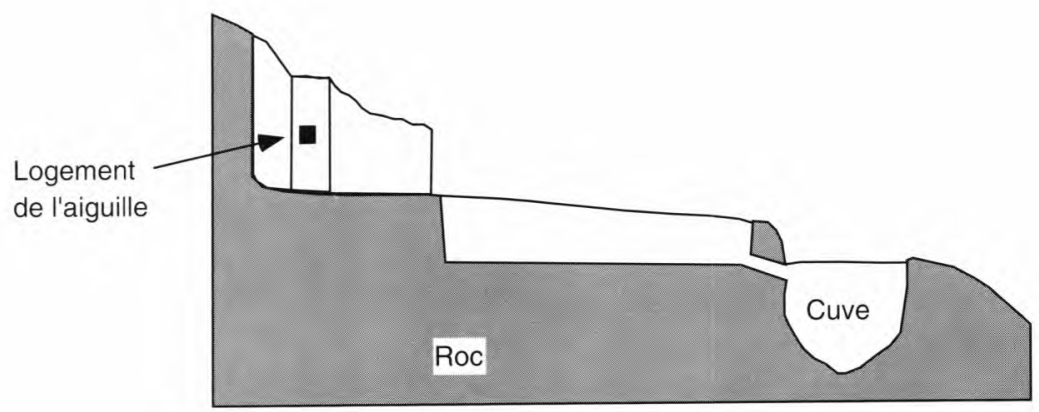

Coupe A-B

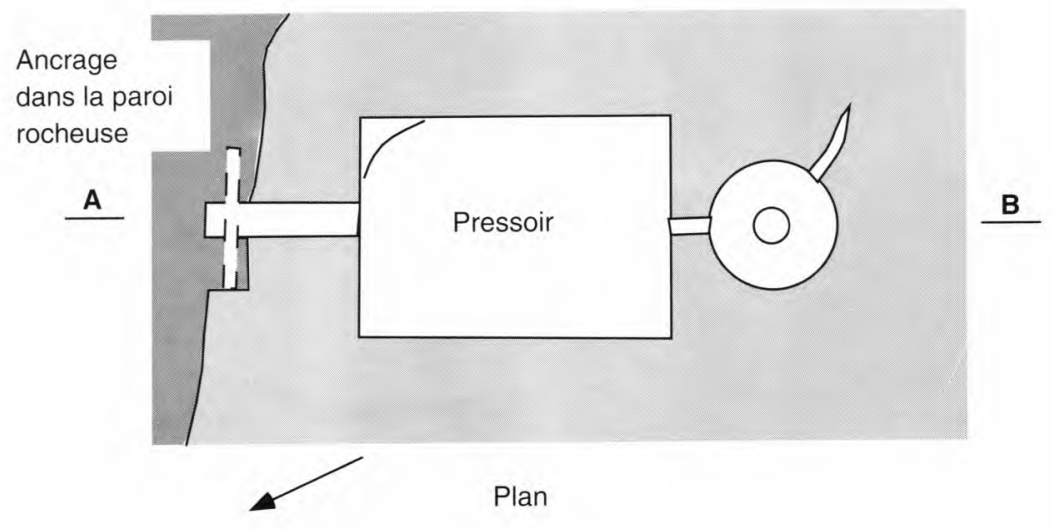

$0 \quad 1 \mathrm{~m}$

FIG. 12 - Pressoir de Sobões da Mina, d'après Russell Cortez 1951, figg. 23. 

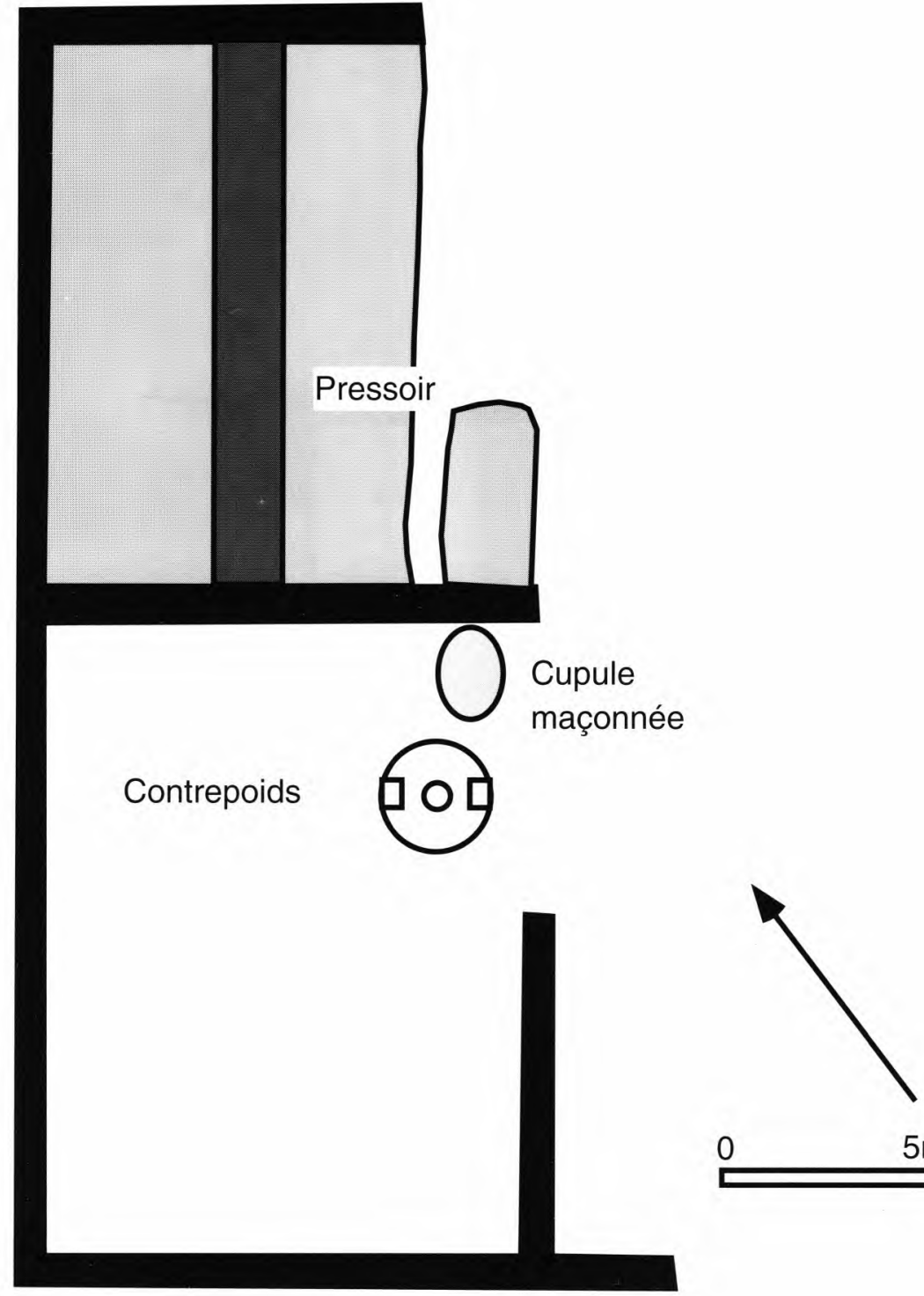

0

$5 \mathrm{~m}$

FIG. 13 - Villa de Monte do Meio, d'après Viana 1957, fig. 3. 

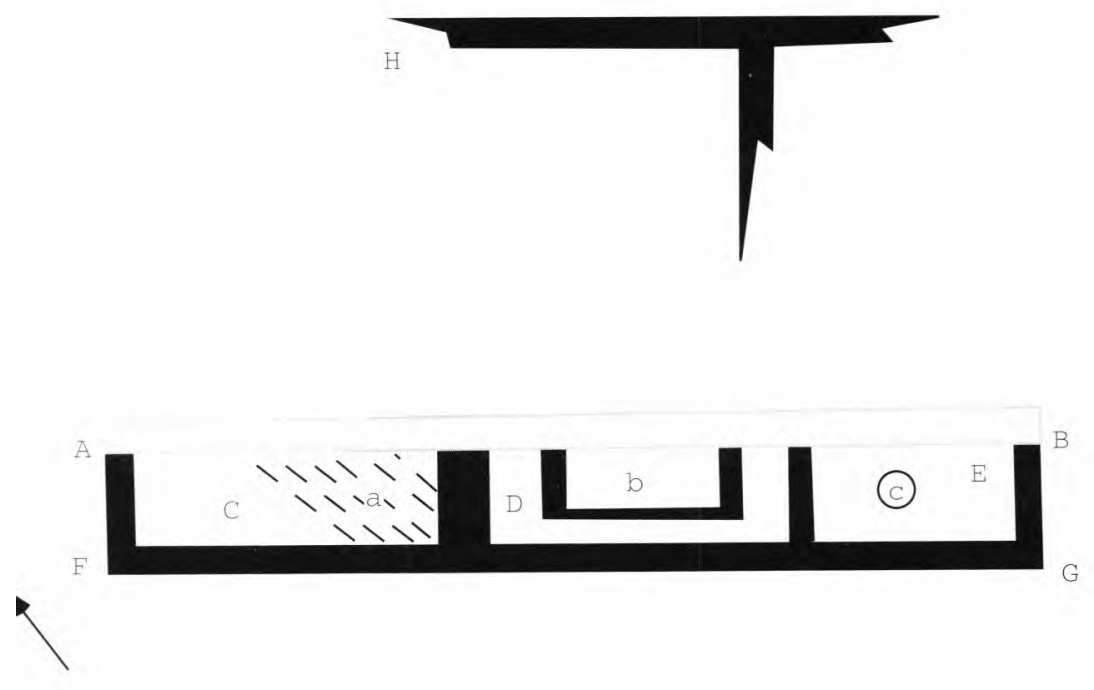

FIG. 14 Villa de Santa Vitoria do Ameixial, d'après Chaves. 


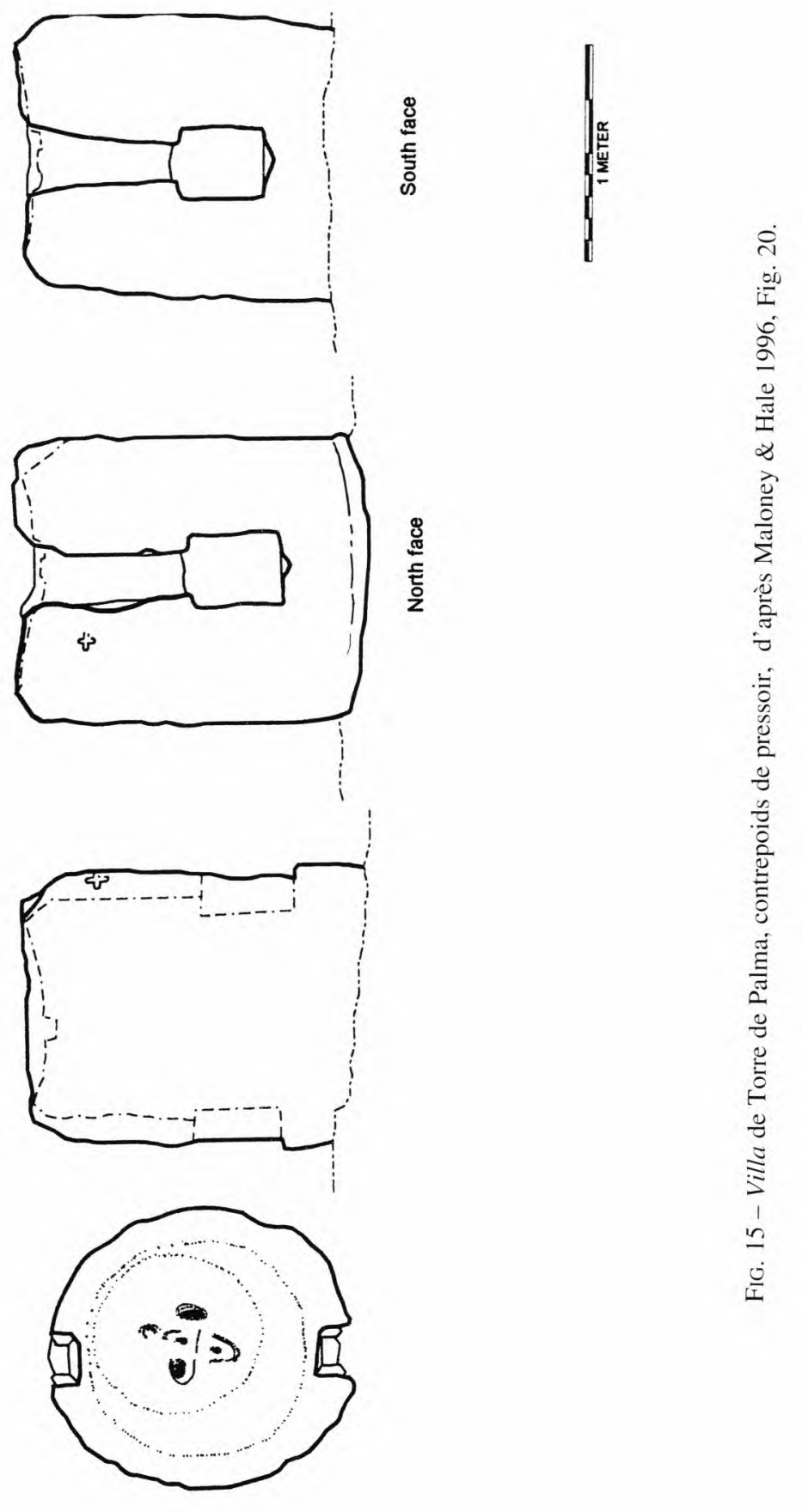




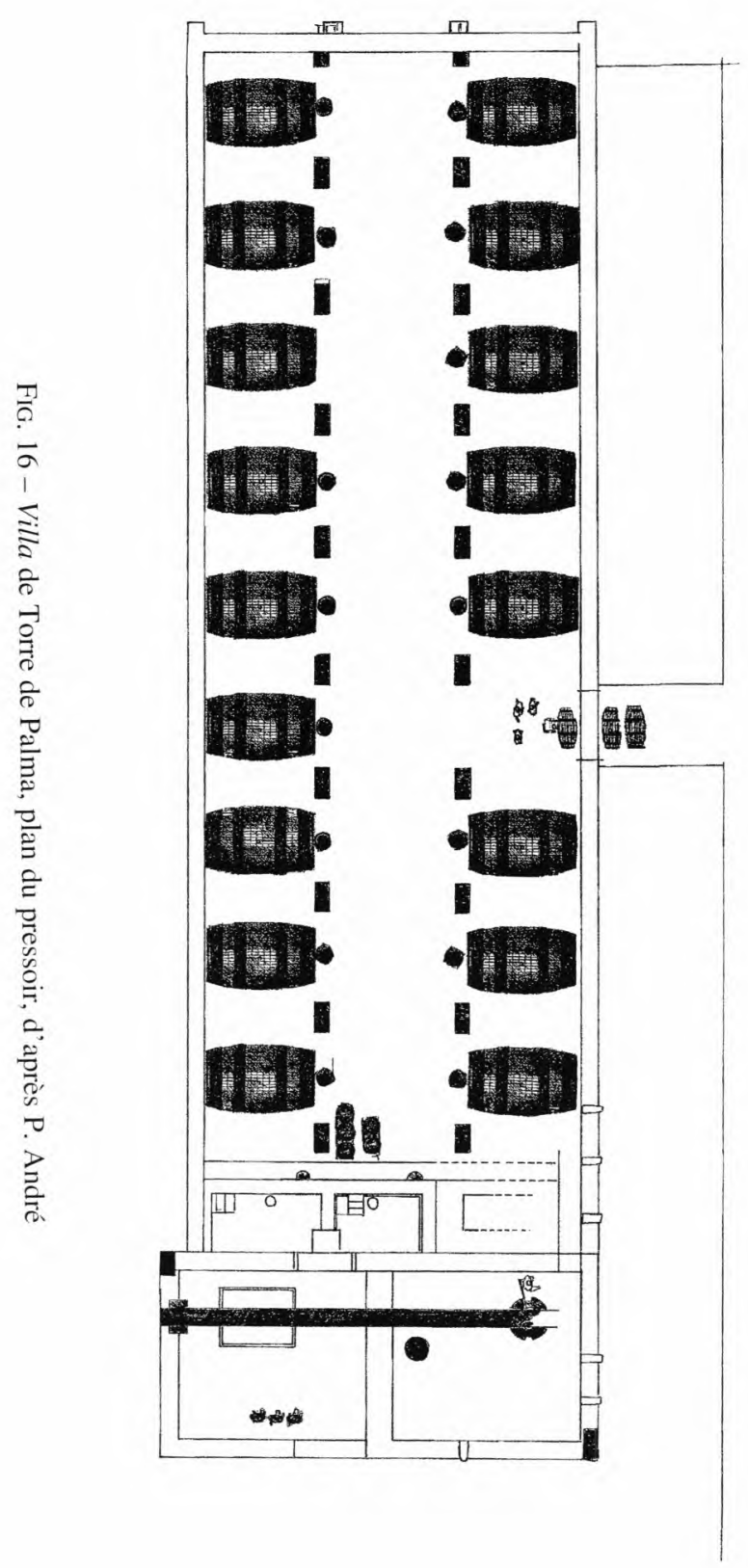

